\title{
Information Aggregation in Auctions with an Unknown Number of Bidders
}

\author{
Ronald M. Harstad ${ }^{\mathrm{a}, *}$ Aleksandar Pekeč ${ }^{\mathrm{b}} \quad$ Ilia Tsetlin ${ }^{\mathrm{c}}$ \\ a Economics Department, University of Missouri, Columbia, MO 65211, USA
}

b The Fuqua School of Business, Duke University, Durham, NC 27708-0120, USA

c INSEAD, 1 Ayer Rajah Avenue, 138676 Singapore

December 27, 2005

* Corresponding author. Phone: +1 573 8823299. Fax:+ 15738823406.

E-mail addresses: $\quad$ harstadr@missouri.edu (R.Harstad), pekec@duke.edu (A. Pekeč), ilia.tsetlin@insead.edu (I. Tsetlin) 


\begin{abstract}
Information aggregation, a key concern for uniform-price, common-value auctions with many bidders, has been characterized in models where bidders know exactly how many rivals they face. A model allowing for uncertainty over the number of bidders is essential for capturing a critical condition for information to aggregate: as the numbers of winning and losing bidders grow large, information aggregates if and only if uncertainty about the fraction of winning bidders vanishes. It is possible for the seller to impart this information by precommitting to a specified fraction of winning bidders, via a proportional selling policy. Intuitively, this makes the proportion of winners known, and thus provides all the information that bidders need to make winner's curse corrections.
\end{abstract}

KEYWORDS: information aggregation, common-value auctions, uncertain level of competition

J.E.L. classifications: D44, D82, D41 


\section{Introduction}

The uniform-price, common-value auction has become the principal game-theoretic model of competitive price formation in economies where information related to the values of goods and services is diversely held. An important concern for a market mechanism is its ability to aggregate private information as the number of market participants becomes large. Wilson (1977) and Milgrom (1979) demonstrate that a single-object auction may aggregate information: the auction price may converge to the unknown true value of the object as the number of bidders grows. Information aggregates under stringent conditions on the potential informativeness of a bidder's signal. Pesendorfer and Swinkels (1997) replace these statistical requirements with two simple economic conditions: they consider a sequence of uniform-price, common-value auctions (each auction with $k$ objects, $n$ bidders, each bidder demanding one object), and show that the unique symmetric equilibrium aggregates information if and only if the number of winners, $k$, and the number of losers, $n-k$, both grow to infinity. ${ }^{1}$ They argue that studying symmetric equilibria along such a sequence of auctions is a reasonable analogy for studying information aggregation in large economies.

An implicit assumption in auction models studying information aggregation is that the number of bidders is common knowledge. It seems unrealistic, however, to suppose that each bidder knows the exact number of rivals in sealed-bid auctions, especially when studying large economies. It is common in sealed-bid auctions for the number of bids submitted to be new information at the time that bids are opened. ${ }^{2}$ An uncertain number of bidders is more likely when the prospect of dozens or hundreds of competitors arises. Electronic call markets face bidders with this uncertainty.

A natural question is whether the same information aggregation conditions extend to a setting where the number of bidders is exogenously random: is common knowledge that the number of winners and the number of losers both surely grow to infinity still sufficient for information aggregation?

The main insight of this paper is that information aggregation is not automatic when bidders in large auctions may not know exactly how many rivals they face. Controlling for uncertainty about the level of competition turns out to be critical. We show that Pesendorfer and Swinkels' (1997) conditions, that the numbers of winning and losing bidders grow to

\footnotetext{
${ }^{1}$ We model the case of a single seller with variable supply selling to multiple competing buyers. All results have complete analogues in the situation with a single buyer with variable demand buying from multiple competing sellers.

${ }^{2}$ For example, the number of bidders in offshore oil auctions, despite being small, is an important uncertainty. In the mid-1980's, a major oil company approached one of the authors about building a model to predict the number of bidders.
} 
infinity, are no longer sufficient to aggregate information. An additional critical condition is that uncertainty about the proportion of winners vanishes, that is, the success ratio $s=$ $k / n$ (the proportion of winners) must be known for information to aggregate. This critical distinction between bidders knowing the numbers of bidders and objects sold, versus merely knowing the success ratio, can only be grasped in a model of the type presented below: we consider a sequence of auctions, with an exogenous distribution of the number of bidders for each element of the sequence. ${ }^{3}$ Moreover, this distinction helps to clarify what causes information aggregation. Were $n$ and $k$ to be fixed (e.g., as in Pesendorfer and Swinkels, 1997) rather than stochastic at each element of the sequence, we could not study the impact of this uncertainty on information aggregation, let alone address the question of whether bidders need to know the number of rivals or merely know the fraction of winning bidders.

We show that the increasing equilibrium bid function (when it exists) is a weighted average of the equilibrium bid functions for the case where $n$ is known. Critically, when the supply/demand ratio is bounded away from zero and one, each of these bid functions is asymptotically a function solely of the success ratio $s=k / n$ (a bidder observing signal $x_{0}$ comes to bid that value $v$ for which the probability of observing a signal $X \geq x_{0}$ equals $s$ ). Thus, if the possible success ratios related to different realizations of the number of bidders approach a common limit, then bidders bid (approximately) as if they knew the number of bidders, and information aggregates. On the other hand, if these success ratios remain distinct, bidders bid significantly differently (from the case with a known number of bidders) and information aggregation cannot be attained. In particular, information aggregates only when the sequence of ratios of the largest possible to the smallest possible number of bidders (in each auction along the sequence) converges to one. In other words, information aggregates only if uncertainty over the number of bidders becomes negligible.

From the seller's perspective, the stochastic process that governs the number of bidders is out of his control (since bidders' participation is exogenous). However, if the seller could vary the quantity sold, ${ }^{4}$ he could adjust it in accordance with the number of bids submitted (once bids are submitted, the seller does not face uncertainty as to the number of submissions); for example, the seller could precommit to sell $k_{i}$ objects if $n_{i}$ bids are submitted. Regardless of its practical implications and feasibility, this more general setting highlights a crucial condition for information aggregation: at the moment of bid submission, bidders do not have to know the number of bidders or the number of objects; however, for information to

\footnotetext{
3This exogenous randomness is specified as in Matthews' (1987) model of private-values auctions and Harstad et al.'s (1990) model of common-value auctions. Both of those papers considered only a singleobject setting with independently drawn private information.

${ }^{4}$ For example, this is possible in auctions of financial instruments (e.g., stocks, bonds, T-bills, et al.).
} 
aggregate, they must know the proportion of winners. Hence, when uncertainty about the number of bidders remains asymptotically significant, information aggregation is attained only if the seller commits to proportional selling.

This raises the question of whether proportional selling serves the expected-revenuemaximization goal of a risk-neutral seller. As information aggregation leads bidders' individualrationality constraints to converge to becoming binding, asymptotically a seller can do no better; the example in the next section indicates he can do notably worse (even in the limit). Under stricter distributional assumptions, Appendix A demonstrates that common-value auctions are weakly asymptotically extractive if and only if proportional selling is used. This is a stronger sense in which information aggregation is in seller's best interests: any other sales mechanism exhibits an unbounded expected revenue shortfall relative to proportional selling.

After a general model definition and characterization of an increasing symmetric equilibrium, Section 3 establishes necessary and sufficient conditions for information aggregation. Section 4 discusses some generalizations and Section 5 concludes. All proofs are in Appendices B-D.

\section{An Illustrative Example}

The value of any one of the identical objects sold at auction is a random variable $V$; each bidder $i$ privately observes an estimate $X_{i}$. For this example, let the common value $V$ be uniform on $[0 ; \bar{v}], \bar{v}>1$, and the conditional estimate density for all $X_{i}$ be uniform on $[v-1 / 2 ; v+1 / 2]$. For simplicity of the illustration, we ignore bidding outside $\digamma=$ $[1 / 2, \bar{v}-1 / 2]$; theorems below show that results are unaffected. ${ }^{5}$

The first case fits the Pesendorfer and Swinkels (1997) assumptions. Consider a sequence of auctions $\mathcal{A}_{1}, \mathcal{A}_{2}, \ldots$; in auction $\mathcal{A}_{k}$, it is common knowledge that $k$ objects are sold to $n=4 k$ bidders. The symmetric equilibrium bid is then $b^{*} 4 k "(x)=x+k / n-1 / 2=x-1 / 4$, $x \in \digamma^{6}{ }^{6}$ This leads directly to information aggregation, as the distribution of the pivotal bidder's signal asymptotically collapses to $x=v+1 / 2-k / n=v+1 / 4$.

Second, consider a sequence of auctions $\mathcal{A}_{1}, \mathcal{A}_{2}, \ldots$; in auction $\mathcal{A}_{k}$, it is common knowledge that $k$ objects are sold, and that the number of bidders $n$ is either $4 k$ or $8 k$, with equal

\footnotetext{
${ }^{5}$ For sizable $\bar{v}, \digamma$ is nearly the entire support of $X_{i}$.

${ }^{6}$ The feature that, for all $n, k, b^{*}$ depends on $k$ and $n$ only through their ratio is a pathology of the uniform distibutions in this example. Lemma 4.1 below obtains a corresponding characterization asymptotically for general distributions.
} 
probabilities. If a bidder knew $n$, he would bid $b^{*} 4 k$ " $(x)=x-1 / 4$ for $n=4 k$, and $b_{\text {" } 8 k}^{*}$ " $(x)=$ $x-3 / 8$ for $n=8 k$, again yielding information aggregation. However, not knowing whether $4 k$ or $8 k$ bidders are present, Theorem 3.1 below shows the symmetric equilibrium in this example is to bid based on the average number of bidders, $6 k$, yielding the equilibrium bid function $b^{*}(x)=x-1 / 3$. Despite satisfying the Pesendorfer/Swinkels conditions (that the numbers of winners and losers both go to infinity), information cannot aggregate in this second case: the distribution of the pivotal bidder's signal asymptotically collapses to $\{v+1 / 4, v+3 / 8\}$, each with probability $1 / 2$. The asymptotic price will be $\{v-1 / 12, v+1 / 24\}$, equally likely.

Third, consider the same sequence of auctions as the second case, except now allow the seller to use proportional selling. One example would be for the seller in auction $\mathcal{A}_{k}$ to precommit to a policy of counting the number of bids submitted, and selling $k$ objects if he counts $4 k$ bids, but $2 k$ objects if there are $8 k$ bids. This policy leads to bidders knowing neither $n$ nor $k$, but knowing their chance of success, $s=k / n=k / 4 k=2 k / 8 k=1 / 4$. Applying Theorem 3.1 , the equilibrium bid in this case depends on the means $\bar{k}$ and $\bar{n}: b^{*}(x)=x+\bar{k} / \bar{n}-1 / 2=$ $x+1.5 k / 6 k-1 / 2=x-1 / 4$. Proportional selling leads to information aggregation: the distribution of the pivotal bidder's signal asymptotically collapses to $\{v+1 / 4, v+2 / 8\}$, each with probability $1 / 2$. In this example, then, knowledge of the number of bidders is not critical to information aggregation, but knowledge of the success ratio $s$ is critical. Below we develop the apparatus necessary to show that this example is in no way special.

\section{A General Model of Stochastic Competition}

This model generalizes Milgrom's (1981) multi-unit common-value auction model with unit demands, by allowing the exact number of bidders to be unknown at the moment of bid submission. As in Milgrom's model, all auctions considered here are sealed-bid auctions with highest-rejected-bid pricing: $k$ homogeneous objects are sold to the $k$ highest bidders at a uniform price equal to the $(k+1)^{\text {st }}$-highest bid, ties broken randomly.

Bidders are drawn from a pool of $N \leq \infty$ potential bidders in accordance with an exogenous symmetric stochastic process, $\Omega=\left\{\left(n_{1}, \pi_{1}\right), \ldots,\left(n_{M}, \pi_{M}\right)\right\}$, specifying that $n_{i}$ bidders are present with probability $\pi_{i}$. The $n_{i}$ are labeled ascendingly: $n_{i}<n_{i^{\prime}}$ for $i<i^{\prime}$. Let $\bar{n}=\sum_{i=1}^{M} \pi_{i} n_{i}$, the expected number of bidders. If the seller commits to a policy $\mathbf{k}=\left\{k_{1}, \ldots, k_{M}\right\}$ to sell $k_{i}$ items if $n_{i}$ bids are submitted, $k_{i}<n_{i}, \forall i$, the resulting stochastic process is

$$
\Omega_{\mathbf{k}}=\left\{\left(n_{1}, k_{1}, \pi_{1}\right), \ldots,\left(n_{M}, k_{M}, \pi_{M}\right)\right\}
$$


As usual, $\Omega_{\mathbf{k}}$ is assumed to be common knowledge. ${ }^{7}$ Note that the policy $\mathbf{k}$ is a deterministic policy choice by seller, but that it acquires its determinism only after bids are submitted; at the time of bidding, it serves to make $(k, n)$ a stochastic pair from the standpoint of a bidder. $^{8}$

The usual case with known $k$ objects and $n$ bidders corresponds to $\Omega_{\mathbf{k}}=\{(n, k, 1)\}$. If the number of objects is fixed at $k$, but the number of bidders is unknown, then $\Omega_{\mathbf{k}}=$ $\left\{\left(n_{1}, k, \pi_{1}\right), \ldots,\left(n_{M}, k, \pi_{M}\right)\right\}$. Finally, distinct $k_{i}$ could correspond to different $n_{i}$.

For a given $k_{i}$ and $n_{i}$, the fraction of winning bidders is called the success ratio, $s_{i}=k_{i} / n_{i}$. The policy $\Omega_{\mathbf{k}}$ represents proportional selling if there is (almost) no uncertainty about the success ratio, i.e., if all success ratios $s_{i}=k_{i} / n_{i}, i=1, \ldots, M$, are approximately equal. ${ }^{9}$

The seller and all potential bidders are risk-neutral. The value of any one of the identical objects is a random variable $V$ with twice differentiable probability density function $g$. Let the support of $g$ be convex, i.e., $g(v)>0$ if $v \in[\underline{v} ; \bar{v}],-\infty \leq \underline{v}<\bar{v} \leq \infty$, and $g(v)=0$ otherwise. Bidder $j$ observes a signal $X_{j}$, a random scalar distributed with cumulative distribution function $F(x \mid v)$ and density $f(x \mid v)$ conditional on $V=v$, where $f$ is assumed to have a third derivative with respect to $v$, and is continuous in $x$.

We assume that $f$ satisfies the Strict Monotone Likelihood Ratio Property (SMLRP): ${ }^{10}$

$$
\frac{f(x \mid v)}{f\left(x \mid v^{\prime}\right)}>\frac{f\left(x^{\prime} \mid v\right)}{f\left(x^{\prime} \mid v^{\prime}\right)} \forall x>x^{\prime}, v>v^{\prime} .
$$

Because of SMLRP, $F(x \mid v)$ is decreasing in $v$. A slight technical strengthening of this assumption, adding that $\partial F(x \mid v) / \partial v<0$ for all $x, v$ such that $0<F(x \mid v)<1$, will be used in the proof of Lemma 4.1.11

Symmetry allows focusing on realized bidder 1 . Bidder 1 is said to be pivotal if his bid is on the boundary between winning and losing. Let the pivotal rival to bidder 1 be the rival

\footnotetext{
${ }^{7}$ This model of exogenous bidders' participation is standard: the single-object case $k_{1}=\ldots=k_{M}=1$ has been studied in Matthews (1987) and Harstad et al. (1990).

${ }^{8}$ We treat the seller as able to demonstrate ex post to suspicious bidders the number of bids submitted, and thus for such a deterministic policy to be credible. In contrast, a seller who stated that "if $n_{i}$ bids are submitted, then I will sell $k_{i 1}$ objects with probability $\vartheta$ and $k_{i 2}$ objects with probability $1-\vartheta$ " would have no way of demonstrating ex post that the (allegedly) committed policy had in fact been applied.

${ }^{9}$ Since $k_{i}$ and $n_{i}$ are integers, only approximate equality is attainable. However, for large $n_{i}$ this integer constraint becomes negligible.

${ }^{10}$ Milgrom (1981) makes the same assumption, and Pesendorfer and Swinkels (1997) assume MLRP. Information aggregation results in Wilson (1977) and Milgrom (1979) rely on much stronger assumptions (see the discussion in Pesendorfer and Swinkels, 1997). On well-behaved domains, the assumption that $v$ and the $x$ 's are affiliated is equivalent up to the interpretation of strictness; cf. Milgrom and Weber (1982), pp. 1098-1101 and 1118-1121. Riley (1988) explains that the assumption that the $x$ 's are strictly stochastically ordered by $v$ is virtually indistinguishable from SMLRP.

${ }^{11}$ Hong and Shum (2004, Corollary 1) require the same strengthening.
} 
bidder with the $k_{i}^{\text {th }}$-highest signal, of the $n_{i}-1$ rivals. Among all $n_{i}$ bidders, the price-setter is the bidder submitting the $\left(k_{i}+1\right)^{\text {st }}$-highest bid. Given $\Omega_{\mathbf{k}}$, the identities of these order statistics may be stochastic, but are well-defined.

An auction is defined by $\mathcal{A}=\left\{\Omega_{\mathrm{k}}, g(\cdot), f(\cdot)\right\}$, specifying the stochastic structure of bidders and objects sold, the prior distribution of the common value, and the conditional distribution of any one bidder's signal.

\subsection{Symmetric equilibrium}

This subsection characterizes a symmetric equilibrium in auction $\mathcal{A}=\left\{\Omega_{\mathbf{k}}, g(\cdot), f(\cdot)\right\}$. Initially, consider the case of a known number of bidders $(n)$ and objects $(k)$, which corresponds to $\Omega_{\mathbf{k}}=\{(n, k, 1)\}$. Let $v_{n k}$ denote the usual conditional value function

$$
v_{n k}(x, y)=E\left[V \mid X_{1}=x, Y_{n-1}^{k}=y\right]
$$

where $Y_{n-1}^{k}$ is the $k^{\text {th }}$-highest of $n-1$ signals. Then

$$
b_{n k}(x)=v_{n k}(x, x),
$$

the expected value given that bidder 1 is pivotal in an $n$-bidder auction for $k$ objects, is the unique symmetric equilibrium bid function (Milgrom (1981), Pesendorfer and Swinkels (1997)).

Theorem 3.1 considers a bidding function for an unknown number of bidders that is a weighted average of the bidding functions for each $\left(n_{i}, k_{i}\right)$ pair. The weights are Bayesian updatings of the probabilities of $n_{i}$ bidders and $k_{i}$ objects (the $i^{\text {th }}$ component of $\Omega_{\mathbf{k}}$ ) under the assumption that bidder 1 is pivotal. Denote

$$
w_{i}(x)=\frac{\frac{\pi_{i} n_{i}\left(n_{i}-1\right) !}{\left(k_{i}-1\right) !\left(n_{i}-k_{i}-1\right) !} \int_{\underline{v}}^{\bar{v}} f^{2}(x \mid t) F^{n_{i}-k_{i}-1}(x \mid t)(1-F(x \mid t))^{k_{i}-1} g(t) d t}{\sum_{j=1}^{M} \frac{\pi_{j} n_{j}\left(n_{j}-1\right) !}{\left(k_{j}-1\right) !\left(n_{j}-k_{j}-1\right) !} \int_{\underline{v}}^{\bar{v}} f^{2}(x \mid t) F^{n_{j}-k_{j}-1}(x \mid t)(1-F(x \mid t))^{k_{j}-1} g(t) d t} .
$$

Theorem 3.1 If there exists a symmetric equilibrium $b^{*}: \Re \rightarrow \Re$ in increasing strategies for $\mathcal{A}=\left\{\Omega_{\mathbf{k}}, g(\cdot), f(\cdot)\right\}$, then it is

$$
b^{*}(x)=\sum_{i=1}^{M} w_{i}(x) b_{n_{i} k_{i}}(x)
$$

where $b_{n_{i} k_{i}}$ and $w_{i}$ are defined by (2) and (3). Conversely, if $b^{*}$ is increasing, then it is the unique symmetric equilibrium in increasing strategies. 
The proof defines a function $v(x, y)$ that is expected value of an object if a bidder observes signal $x$, and his pivotal rival observes signal $y$, given the distributions of the numbers of bidders and objects. The existence of an increasing symmetric equilibrium bid function is shown to imply that $v(x, x)$ is increasing. Given this fact, $b^{*}(x)=v(x, x)$, and any deviation from $v(x, x)$ when rivals all bid $b^{*}$ is then unprofitable. Details of the proof are in Appendix B.

Theorem 3.1 cannot be strengthened to guarantee existence of an increasing symmetric equilibrium. ${ }^{12}$ Example 3.2 finds that existence or non-existence of equilibrium in increasing strategies can be attained simply by changing the degree of uncertainty about the number of bidders.

Example 3.2 Consider an auction for $k=1$ object with $\Omega_{\mathbf{k}}=\left\{(2,1,1 / 2),\left(n_{2}, 1,1 / 2\right)\right\}$, that is, there are either 2 or $n_{2}$ bidders, each equally likely. Let $g(v)$ be uniform on $[0 ; \bar{v}]$, $\bar{v}>1$, and let $f(x \mid v)$ be uniform on $[v-1 / 2 ; v+1 / 2]$. Note that uniform distribution does not satisfy SMLRP (eq. (1)), ${ }^{13}$ but the proof of Theorem 3.1 is valid for this distribution as well, because property (13) of the posterior distribution is satisfied for $\bar{v}>1$, and therefore the corresponding $v(x, y)$ is increasing in $x$. For $x<1 / 2$, derivation in Appendix $B$ yields:

$$
b^{*}(x)=x+\frac{1}{2}-\frac{\left(x+\frac{1}{2}\right)+\left(n_{2}-1\right)\left(x+\frac{1}{2}\right)^{n_{2}-1}}{2+n_{2}\left(x+\frac{1}{2}\right)^{n_{2}-2}} .
$$

As $x \nearrow 1 / 2$, i.e., for $x$ close enough to but less than $1 / 2$, this function is increasing for $n_{2} \leq 5$ and is decreasing for $n_{2} \geq 6$. Therefore, by Theorem 3.1, an increasing symmetric equilibrium exists for $n_{2} \leq 5$, but no such equilibrium exists if $n_{2} \geq 6$.

This example uses small values of $n$ and $k$ for computational ease. It is straightforward to generalize the example and construct a sequence of auctions, with numbers of winners and losers surely going to infinity, and $s_{i}$ 's bounded away from 0 and 1 , but no increasing symmetric equilibrium for any element of the sequence. ${ }^{14}$ In conclusion, it appears that an increasing symmetric equilibrium will exist for any $\Omega_{\mathbf{k}}$ in which the variability of $n$ and $k$ is

\footnotetext{
${ }^{12}$ While $v_{n k}(x, y)$ is increasing in both arguments, $v(x, y)$ is increasing in $x$ but sufficient variability in the uncertain number of bidders will prevent it from being increasing in $y$.

${ }^{13}$ However, the uniform distribution satisfies MLRP and might be viewed as a limit as $a \rightarrow 0$ of the following distributions on $[v-1 / 2 ; v+1 / 2]: f_{a}(x \mid v)=1-a(x-v)^{2}+a / 12$. For any $6 \geq a>0, f_{a}(x \mid v)$ satisfies SMLRP (1).

${ }^{14}$ Theorem 3.1 is necessarily weaker than the equilibrium existence theorem in Pesendorfer and Swinkels (1997), due to uncertainty over the number of bidders. Remark 8.1 in Appendix A demonstrates that the "loser's curse" on which they rely cannot be extended to this more general model. The critical aspect of their proof is this: if a bidder is told that he is tied for being the last winning bidder, the conditional expectation of $V$ is then increasing in the number of biddders who are tied with him. In this model, if a pivotal bidder is told that he has lost a tie breaker, it is possible that more of a given $n-1$ rivals were tied with him,
} 
not too extreme. Unfortunately, no simple statement of the threshold, between $\Omega_{\mathbf{k}}$ which are too extreme, and nondegenerate $\Omega_{\mathbf{k}}$ for which increasing symmetric equilibria are assured to exist, can be found.

\section{Information Aggregation}

Information aggregation and other asymptotic properties will be investigated on sequences of auctions $\mathcal{A}_{1}, \mathcal{A}_{2}, \ldots$, denoted by $\left\{\mathcal{A}_{\zeta}\right\}_{\zeta=1,2, \ldots}$, along which the numbers of bidders and objects grow large while, for expositional clarity, keeping the probability vector $\left(\pi_{1}, \ldots, \pi_{M}\right)$, common value distribution, and bidders' signal distribution fixed. Thus, all sequences of auctions $\left\{\mathcal{A}_{\zeta}\right\}$ considered below satisfy: ${ }^{15}$

$$
\begin{aligned}
& \text { [i] } n_{1_{\zeta}} \rightarrow \infty \text { as } \zeta \rightarrow \infty \\
& \text { [ii] } \pi_{i_{\zeta}}=\pi_{i}>0 \text { for all } i=1, \ldots, M \text {, all } \zeta=1,2, \ldots \\
& \text { [iii] } g_{\zeta}(\cdot)=g(\cdot) \text { and } f_{\zeta}(\cdot)=f(\cdot) \text { for all } \zeta=1,2, \ldots
\end{aligned}
$$

\subsection{Asymptotic dependence of $b_{n k}$ on success ratio only}

As shown by (4), $b^{*}$ is a weighted average of the increasing equilibrium bid functions for known numbers of bidders. Thus, information aggregation in the presence of uncertainty over the number of bidders and objects depends on the asymptotic behavior in large auctions of the function $b_{n k},(2)$ above. If $(n, k)$ were known, this function would be the symmetric equilibrium bid function. This subsection shows that $b_{n k}$ comes to depend on $(n, k)$ only through the success ratio $s=k / n$.

The equilibrium bid function for a known number of bidders, $b_{n k}$, is a conditional expectation of $V$, conditioned on a signal $x$ being tied for the $k^{\text {th }}$-order and $(k+1)^{\text {st }}$-order statistics out of $n$ signals. All a bidder would care about, if a large enough (known) number of bids were submitted, is a simpler approximation: the expected value of $V$ conditional on $s=k / n$ being the probability that any single signal is $x$ or larger. Let $\kappa(x, s)$ denote a realization

suggesting a higher expected value. However, it is also possible that a greater number of bidders tied for pivotal bid means no more than an increase in the likelihood of events with a greater number of bidders. Lack of a general relationship between the number of bidders tied for pivotal bid and the number of bids submitted prevents a general existence theorem.

${ }^{15}$ These particular sequences are chosen for ease of exposition only. We discuss in Sections 3.2 and 4 below how the necessary and sufficient conditions apply more generally. Also note that, as shown by Theorem 3.1, bidding strategies do not depend upon $N$, the number of potential bidders. Thus, along a sequence of auctions, $N$ can change in any pattern subject to the constraint $N \geq n_{M_{\zeta}}$. 
for $v$ with the property that the probability of getting a signal of $x$ or greater is exactly $s=k / n$. Thus, $\kappa(x, t)$ is a solution of the equation $1-F(x \mid \kappa(x, t))=t$ if such a solution exists. Further, denoting $\left[\underline{v_{x}}, \overline{v_{x}}\right]$ the support of the posterior distribution of $v$ given $x, \kappa$ is defined for all $0<t<1$ :

$$
\kappa(x, t)= \begin{cases}\underline{v_{x}}, & t<1-F\left(x \mid \underline{v_{x}}\right), \\ \kappa(x, t) \text { such that } 1-F(x \mid \kappa(x, t))=t, & 1-F\left(x \mid \underline{v_{x}}\right) \leq t \leq 1-F\left(x \mid \overline{v_{x}}\right), \\ \overline{v_{x}}, & 1-F\left(x \mid \overline{v_{x}}\right)<t .\end{cases}
$$

To formalize the asymptotic dependence of $b_{n k}(x)$ on the success ratio only, consider a sequence of auctions $\left\{\mathcal{A}_{u}\right\}_{u=1,2, \ldots}$. Along this sequence, $\Omega_{\mathbf{k}_{u}}=\left\{\left(n_{u}, k_{u}, 1\right)\right\} \forall u$ and, by (5), $n_{u} \rightarrow \infty$ as $u \rightarrow \infty$. That is, the numbers of bidders and objects sold are always known, with the number of bidders approaching infinity. The number of objects $k_{u}$ in auction $\mathcal{A}_{u}$, and thus the success ratio $s_{u}$, may take any pattern as long as $0<\liminf s_{u} \leq \lim \sup s_{u}<1$.

The following Lemma states that $b_{n k}(x)$ and $\kappa(x, s)$ are asymptotically equal, i.e., as $n$ gets large, the equilibrium bid $b_{n k}(x)$ converges to the value of $v$ that yields a probability $s$ of observing a signal greater or equal to $x .{ }^{16}$ The standard $O$ notation is used, defined by $\lim \sup _{t \rightarrow 0}|O(t) / t|<\infty$.

Lemma 4.1 Let $\left\{\mathcal{A}_{u}\right\}_{u=1,2, \ldots}$ with $\Omega_{\mathbf{k}_{u}}=\left\{\left(n_{u}, k_{u}, 1\right)\right\}$, such that $0<\lim \inf s_{u} \leq \lim \sup s_{u}<$ 1. Then, for every signal $x$

$$
b_{n_{u} k_{u}}(x)=\kappa\left(x, s_{u}\right)+O\left(\frac{1}{n_{u}}\right) .
$$

\subsection{Aggregation with an unknown number of bidders}

This subsection analyzes necessary and sufficient conditions for information aggregation when there is uncertainty about the number of bidders. It turns out that proportional selling, i.e., keeping the success ratio $s=k_{i} / n_{i}$ constant, is crucial.

Consider a sequence of auctions $\left\{\mathcal{A}_{\zeta}\right\}_{\zeta=1,2, \ldots}$. By (5), $n_{1_{\zeta}} \rightarrow \infty$ as $\zeta \rightarrow \infty$, that is, while the number of bidders remains uncertain, the smallest possible realization of the number of bidders, $n_{1_{\zeta}}$, grows unboundedly large. Also note that whenever the success ratio is bounded away from zero and one, $0<\min _{i} \liminf _{\zeta} s_{i_{\zeta}} \leq \max _{i} \limsup _{\zeta} s_{i_{\zeta}}<1$, Pesendorfer

\footnotetext{
${ }^{16}$ This Lemma demonstrates, as a general result, a clarification of a conjecture, relative to a special example, in Pesendorfer and Swinkels: "Note that in the limit it must be the case that whenever a bid is pivotal then the value of the object is equal to that bid. And for large $n$, the actual number of signals below $x$ will always be very close to $F(x \mid v)$ if the true value is $v . "$ ([1997], pg. 1261, notation in quotation changed to fit this model.) A similar result was independently obtained by Hong and Shum (2004), see their proof of Theorem 2, eq.(A.9).
} 
and Swinkels' conditions for information aggregation when there is no uncertainty about the number of bidders are automatically satisfied (i.e., both the number of winners and the number of losers grow unboundedly). Let $P^{\zeta}$ denote the random (per object) selling price for auction $\mathcal{A}_{\zeta}$. By definition (see, e.g., Pesendorfer and Swinkels, 1997) the sequence of auctions $\left\{\mathcal{A}_{\zeta}\right\}$ aggregates information if

$$
\forall \varepsilon>0 \lim _{\zeta \rightarrow \infty} \operatorname{Pr}\left[\left|P^{\zeta}-V\right|>\varepsilon\right]=0
$$

Theorem 4.2 Consider a sequence of auctions $\left\{\mathcal{A}_{\zeta}\right\}_{\zeta=1,2, \ldots}$ such that $b_{\zeta}^{*}$ is a symmetric equilibrium for each $\mathcal{A}_{\zeta}$. If there exists $s \in(0,1)$ such that $\lim _{\zeta \rightarrow \infty} s_{i_{\zeta}}=s$ for all $i=1, \ldots, M$, then $\left\{\mathcal{A}_{\zeta}\right\}$ aggregates information.

Theorem 4.2 provides a sufficient condition for information aggregation: proportional selling allows every bidder to know the success ratio even if the number of bidders is unknown, and, as shown by Lemma 4.1, a bidder in an auction with a large number of bidders cares only about the success ratio. ${ }^{17}$ In particular, when the number of objects sold is invariant with respect to the number of bidders, information aggregates whenever uncertainty about the number of bidders is negligible:

Corollary 4.3 Let $\left\{\mathcal{A}_{\zeta}\right\}_{\zeta=1,2, \ldots}$ be a sequence of auctions such that $k_{i_{\zeta}}=k_{\zeta}$ for all $i, \zeta$ and such that $b_{\zeta}^{*}$ is a symmetric equilibrium for each $\mathcal{A}_{\zeta}$. Suppose that $0<\liminf \operatorname{in}_{\zeta \rightarrow \infty} k_{\zeta} / n_{M_{\zeta}} \leq$ $\lim \sup _{\zeta \rightarrow \infty} k_{\zeta} / n_{1_{\zeta}}<1$. Then information aggregates if $\lim _{\zeta \rightarrow \infty} n_{M_{\zeta}} / n_{1_{\zeta}}=1$.

Essentially, Theorem 4.2's sufficient condition, proportional selling, is also necessary. If the price-setting bidder is not bidding as if the success ratio is known, then information does not aggregate. Suppose the success ratio cannot be known at least for some signals. That is, suppose there exist $L, U \in\{1, \ldots, M\}$ such that $0<\lim _{\zeta \rightarrow \infty} s_{L_{\zeta}}=s_{L}<s_{U}=\lim _{\zeta \rightarrow \infty} s_{U_{\zeta}}<$ 1 , and there exists $x_{0}$ such that $\underline{v}<\kappa\left(x_{0}, s_{L}\right)<\kappa\left(x_{0}, s_{U}\right)<\bar{v}$. As a result, a price-setting bidder, observing signal $x$ in some neighborhood of $x_{0}$, cannot distinguish between the events $\left\{v=\kappa\left(x_{0}, s_{L_{\zeta}}\right)\right\}$ and $\left\{v=\kappa\left(x_{0}, s_{U_{\zeta}}\right)\right\}$ near the limit as $\zeta \rightarrow \infty$, so his price-setting bid cannot aggregate information in at least one of these events. ${ }^{18}$ The formal statement:

\footnotetext{
${ }^{17}$ Note that if the asymptotic success ratio is $s=0$ or $s=1$, the question of information aggregation depends on further assumptions about model elements (Pesendorfer and Swinkels, 1997; Hong and Shum, 2004).

${ }^{18}$ In the terminology of Milgrom, 1979, pg. 681, eq. (4), the events $\left\{v=\kappa\left(x_{0}, s_{L_{\zeta}}\right)\right\}$ and $\left\{v=\kappa\left(x_{0}, s_{U_{\zeta}}\right)\right\}$ are not distinguishable given the signal $x_{0}$ as $\zeta \rightarrow \infty$.
} 
Theorem 4.4 Consider a sequence of auctions $\left\{\mathcal{A}_{\zeta}\right\}_{\zeta=1,2, \ldots}$ such that $b_{\zeta}^{*}$ is a symmetric equilibrium for each $\mathcal{A}_{\zeta}$. Suppose there exist $L, U \in\{1, \ldots, M\}, s_{L}, s_{U}$ and an infinite subsequence $\left\{\mathcal{A}_{\zeta^{\prime}}\right\}_{\zeta^{\prime}=1,2, \ldots}$ of $\left\{\mathcal{A}_{\zeta}\right\}$ such that $0<\lim _{\zeta^{\prime} \rightarrow \infty} s_{L_{\zeta^{\prime}}}=s_{L}<s_{U}=\lim _{\zeta^{\prime} \rightarrow \infty} s_{U_{\zeta^{\prime}}}<1$, and there exists a signal $x_{0}$ such that $\underline{v}<\kappa\left(x_{0}, s_{L}\right)<\kappa\left(x_{0}, s_{U}\right)<\bar{v}$. Then $\left\{\mathcal{A}_{\zeta}\right\}$ does not aggregate information.

Thus, Theorems 4.2 and 4.4 provide the sense in which proportional selling joins the Pesendorfer-Swinkels "double largeness" as necessary and sufficient conditons for information aggregation. ${ }^{19}$

\section{Possible Generalizations}

Asymptotic properties for large auctions have been analyzed for sequences $\mathcal{A}_{\zeta}$ in which the probability distribution of the numbers of bidders and objects is fixed along the sequence. For necessity, Theorem 4.4, dealing with such constrained sequences suffices. As for sufficiency, Theorem 4.2 readily generalizes if this assumption is relaxed to $\lim _{\zeta \rightarrow \infty} \pi_{i_{\zeta}}=\pi_{i}>0, i=$ $1, \ldots, M$. Furthermore, if the model of stochastic competition is generalized to allow for infinite $M$, Theorem 4.2 extends provided that whenever convergence is required for all $i$ (e.g., $\lim s_{i_{\zeta}}=s$ in Theorem 4.2), it is at a uniform rate.

Our model assumes the number of bidders is independent of $V$, and bidders' signals are independent conditional on $V$. Both these assumptions ease exposition and can be relaxed. First, Theorem 3.1, Theorem 4.2, and Theorem 4.4 readily extend if, for each realization $n_{i}$ of the number of bidders, the common value $V$ is drawn via a different pdf $g_{i}(v)$. Such a description could model more optimistic views about $V$ should a larger number of bidders compete, e.g., $E\left[V \mid n_{U}\right]>E\left[V \mid n_{L}\right] \Leftrightarrow n_{U}>n_{L}$.

Second, the formula for $b^{*}$ remains almost unchanged when conditionally dependent signals are allowed in the following sense. Conditional on a common factor $c$ that is drawn from a density $g(c)$, signals are independently drawn from a density $f(x \mid c)$ that satisfies SMLRP. ${ }^{20}$ The common value $V$ is then drawn from a density $q(v \mid c)$ with support $V_{c}$, and conditional on $c, V$ and signals are independent. Theorem 3.1 extends if $q(v \mid c)$ either satisfies SMLRP or is unbiased (i.e., $E[V \mid c]=c$ ). Note that in this setting a sequence of auctions might

\footnotetext{
${ }^{19}$ The following corollary is roughly parallel to Corollary 4.3: Let $\left\{\mathcal{A}_{\zeta}\right\}_{\zeta=1,2, \ldots}$ be a sequence of auctions such that $k_{i_{\zeta}}=k_{\zeta}$ for all $i, \zeta$ and such that $b_{\zeta}^{*}$ is a symmetric equilibrium for each $\mathcal{A}_{\zeta}$. Suppose $\liminf \operatorname{in}_{\zeta \rightarrow \infty} n_{M_{\zeta}} / n_{1_{\zeta}}>1$, and there exist $L, U \in\{1, \ldots, M\}, s_{L}, s_{U}$ and an infinite subsequence $\left\{\mathcal{A}_{\zeta^{\prime}}\right\}_{\zeta^{\prime}=1,2, \ldots}$ of $\left\{\mathcal{A}_{\zeta}\right\}$ such that $0<\lim _{\zeta^{\prime} \rightarrow \infty} s_{L_{\zeta^{\prime}}}=s_{L}<s_{U}=\lim _{\zeta^{\prime} \rightarrow \infty} s_{U_{\zeta^{\prime}}}<1$, and a signal $x_{0}$ such that $\underline{v}<\kappa\left(x_{0}, s_{L}\right)<\kappa\left(x_{0}, s_{U}\right)<\bar{v}$. Then $\left\{\mathcal{A}_{\zeta}\right\}$ does not aggregate information.

${ }^{20}$ See also Milgrom (1981), Assumption A2* on pg. 938.
} 
aggregate information only about the common factor $c$, but the variance of $V$ conditional on the auction price cannot be lower than the conditional variance of $V$ given $c$.

Combining these two generalizations, let common factor $c$ be drawn from $g_{i}(c)$ with supports $C_{i}$, where different $g_{i}(c)$ correspond to different $n_{i}$. For this combination, the unique candidate for an increasing symmetric equilibrium bid function is

$b^{*}(x)=\frac{\sum_{i=1}^{M} \frac{\pi_{i} n_{i}\left(n_{i}-1\right) !}{\left(k_{i}-1\right) !\left(n_{i}-k_{i}-1\right) !} \int_{C_{i}}\left(\int_{V_{c}} v q(v \mid c) d v\right) f^{2}(x \mid c) F^{n_{i}-k_{i}-1}(x \mid c)(1-F(x \mid c))^{k_{i}-1} g_{i}(c) d c}{\sum_{i=1}^{M} \frac{\pi_{i} n_{i}\left(n_{i}-1\right) !}{\left(k_{i}-1\right) !\left(n_{i}-k_{i}-1\right) !} \int_{C_{i}} f^{2}(x \mid t) F^{n_{i}-k_{i}-1}(x \mid t)(1-F(x \mid t))^{k_{i}-1} g_{i}(t) d t}$.

As mentioned, Theorem 3.1 extends.

Finally, the model's restriction to single-unit demand can be extended somewhat. Consider an auction for $k=m * l$ objects in which every bidder is allowed to obtain no more than $l$ units, and can submit up to $l$ bids, which need not be identical. Then one symmetric equilibrium (there might be others) calls for each bidder to submit $l$ identical bids, each equal to the bid that would be submitted in an auction for $m$ objects with a single-unit purchase restriction. $^{21}$ For example, if there are $2 l$ objects for sale and bidders can bid for $1, \ldots, l$ objects, then a symmetric equilibrium is the following: every bidder submits $l$ identical bids, and the bid function is as if there were two objects for sale.

\section{Conclusions}

In ordinary auctions, a large amount of competition is likely to be accompanied by some degree of uncertainty on the part of bidders as to exactly how many bidders are competing. In the presence of this uncertainty, information does not automatically aggregate. The basic reason for this is not a technical issue over properties of signals, as in Wilson (1977) and Milgrom (1979). Rather it is because a bidder, uncertain as to the extent of rivalry, does not know how much of a winner's curse correction to make. ${ }^{22}$ For a fixed number of objects, the above effect places a bound on the uncertainty about the number of bidders: if the difference between the largest and the smallest numbers of bidders grows unboundedly, but at a slower rate than the (smallest) number of bidders grows, then the conditions of Corollary 4.3 are satisfied and information aggregates.

\footnotetext{
${ }^{21}$ This suggests that, in general, properties of the unit-demand common-value auction models might also be valid in some multi-unit demand settings.

${ }^{22}$ Note that, in the independent private-values setting, the assumption that the number of bidders is known is rather innocuous: bidding strategies directly extend to the case of an unknown number of bidders, and revenue equivalence holds (Matthews, 1987). In the common-value setting, on the contrary, this assumption significantly changes the picture of symmetric equilibrium bidding strategies and information aggregation properties.
} 
The analysis presented here demonstrates that in large auctions bidders do not have to know the number of bidders and the number of objects, but they have to know the success ratio (proportion of winners). Thus a proportional selling policy yields information aggregation. That is, by committing to vary the number of objects sold proportionally to the number of bids submitted, the seller can implicitly let bidders know the success ratio when they (and he) are still uncertain about the number of bidders. Proportional selling is also necessary, in that any systematic deviation from it results in a failure of information aggregation.

Beyond technical issues, these results place a different philosophical bent on the efficiency of competitive markets. Prior papers made information aggregation a function of statistical properties of signals (Wilson, 1977 and Milgrom, 1979), or of the extent of competition (Pesendorfer and Swinkels, 1997). In either case, information either aggregated automatically, or automatically failed to aggregate. The recognition here that bidders may face non-negligible uncertainty over the number of rivals makes information aggregation depend on whether proportional selling is used, and thus makes it an issue of selling policy.

\section{Acknowledgments.}

We thank a diligent associate editor and two conscientious referees for valuable suggestions. 


\section{Appendix A: Asymptotic Extraction}

Information aggregation is per se more useful to the analyst than to the seller: even if the price per unit converges to the true value, the number of objects being sold could converge to infinity faster than the convergence of the price. Hence the seller may be left with an infinite shortfall of total revenue below the total value of objects sold.

Total expected profit (summed over potential bidders) in auction $\mathcal{A}=\left\{\Omega_{\mathbf{k}}, g(\cdot), f(\cdot)\right\}$ is:

$$
S\left(\Omega_{\mathbf{k}}\right)=\sum_{i=1}^{M} \pi_{i} k_{i} E\left[V-P_{i}\right] ;
$$

in this expression $P_{i}$ denotes the (per-object) price conditional on the realization of the $i^{\text {th }}$ element of $\Omega_{\mathbf{k}}$ when all bidders use $b^{*}$ (eq. (4)). ${ }^{23}$

A sequence $\left\{\mathcal{A}_{\zeta}\right\}_{\zeta=1,2, \ldots}$ attains full asymptotic extraction if $\lim _{\zeta \rightarrow \infty} S\left(\Omega_{\mathbf{k}_{\zeta}}\right)=0$. If the number of objects is bounded, i.e., $\lim _{\sup _{\zeta \rightarrow \infty}} k_{i_{\zeta}}<\infty$ for every $i$, asymptotic extraction coincides with asymptotic efficiency. By asymptotic efficiency is meant that objects will be sold whenever the common value exceeds the seller's valuation. Thus, if the seller's valuation is $v-\varepsilon$, asymptotic efficiency requires that the expected auction price converges to $v$ (Pesendorfer and Swinkels, 1997, pg. 1265). ${ }^{24}$

If the number of objects is unbounded, i.e., for some $i, \lim \sup _{\zeta \rightarrow \infty} k_{i_{\zeta}}=\infty$, asymptotic extraction is stronger than asymptotic efficiency: along an asymptotically efficient sequence of auctions, the total expected profit could be bounded above zero or even grow unboundedly. A sequence of auctions is weakly asymptotically extractive if $\lim \sup _{\zeta \rightarrow \infty} S\left(\Omega_{\mathbf{k}_{\zeta}}\right)<\infty$. Weak asymptotic extraction guarantees that the total expected profit is bounded and, for an unbounded number of objects, is a stronger property than asymptotic efficiency. Furthermore, (weak) asymptotic extraction is not nested with respect to information aggregation, because the latter requires that the variance of the difference between the auction price and $v$ vanishes.

The importance of eliminating any uncertainty about the success ratio, by selling proportionally, extends beyond concern about information aggregation. This appendix demonstrates that, under the following two assumptions, a sequence of auctions $\left\{\mathcal{A}_{\zeta}\right\}_{\zeta=1,2, \ldots}$ is weakly asymptotically extractive if and only if $\Omega_{\mathbf{k}_{\zeta}}$ satisfies proportional selling.

\footnotetext{
${ }^{23} \mathrm{An}$ individual participating bidder's expected profit is $\sum_{i=1}^{M}\left(\pi_{i} n_{i} / \bar{n}\right)\left(k_{i} / n_{i}\right) E\left[V-P_{i}\right]=S\left(\Omega_{\mathbf{k}}\right) / \bar{n}$, where $\bar{n}=\sum_{i=1}^{M} \pi_{i} n_{i}$, since $\pi_{i} n_{i} / \bar{n}$ is the probability of $n_{i}$ bidders given that the bidder participates in the auction, and $E\left[V-P_{i}\right]$ is the expected profit in the event of winning and this event has (ex-ante) probability $k_{i} / n_{i}$, conditioned on $n_{i}$ bidders, and is ex-ante in that it is not conditioned on a realization of a bidder's signal.

${ }^{24}$ Note that asymptotic efficiency is a weaker property than information aggregation: the former only requires convergence of the expected price, while the latter requires zero variance of $(P-V)$. Several such useful distinctions are in Kremer (2002).
} 
Assumption 7.1 Location invariance: the pdf of conditional density of signals $f$ is locationinvariant, i.e., has the form $f(x \mid v)=h(x-v)$, with the corresponding $c d f F(x \mid v)=H(x-v)$.

Assumption 7.2 Diffuse prior: the distribution of the common value $v$ is diffuse relative to the signal distribution, i.e., the posterior density of $v$ given $x$ is $g(v \mid x)=f(x \mid v) / \int_{-\infty}^{\infty} f(x \mid t) d t$.

Assumption 7.2 is equivalent to assuming an improper density $g(v) \sim$ const on the whole real line. Alternatively, an improper diffuse prior distribution might be viewed as a limit of a normal distribution as its variance goes to infinity. Assumptions 7.1 and 7.2 are used and discussed in, e.g., Klemperer (1999) and Winkler and Brooks (1980).

Corollary 7.3 Under Assumptions 7.1 and 7.2, $b^{*}$ is increasing in $x$ for any $\Omega_{\mathrm{k}}$, so by Theorem 3.1, it is the unique increasing symmetric equilibrium.

Theorem 7.4 Let Assumptions 7.1 and 7.2 hold for a sequence of auctions $\left\{\mathcal{A}_{\zeta}\right\}_{\zeta=1,2, \ldots}$

$[i]$ Assume that there exists $\mu \in(1, \infty)$ such that $n_{M_{\zeta}} / n_{1_{\zeta}}<\mu \forall \zeta$. If there exists $s_{0} \in(0,1)$ and $\omega \in(0, \infty)$ such that $\left|\left(s_{i_{\zeta}}-s_{0}\right) n_{1_{\zeta}}\right|<\omega \forall i, \zeta$, then

$$
\limsup _{\zeta \rightarrow \infty} S\left(\Omega_{\mathbf{k}_{\zeta}}\right)<\infty
$$

[ii] If there exists a subsequence $\left\{\mathcal{A}_{\zeta^{\prime}}\right\}, L, U, s_{L}$, and $s_{U}$, such that $0<\lim _{\zeta^{\prime} \rightarrow \infty} s_{L_{\zeta^{\prime}}}=s_{L}<$ $s_{U}=\lim _{\zeta^{\prime} \rightarrow \infty} s_{U_{\zeta^{\prime}}}<1$, then

$$
\limsup _{\zeta \rightarrow \infty} S\left(\Omega_{\mathbf{k}_{\zeta}}\right)=\infty
$$

Note that $[i]$ assumes that the relative uncertainty over the number of bidders does not grow unboundedly as the average number of bidders grows (since $n_{M_{\zeta}} / n_{1_{\zeta}}<\mu \forall \zeta$ ), and that, for every $i, s_{i_{\zeta}}$ converges to $s_{0}$ as fast as $1 / n_{1_{\zeta}}$ converges to zero (since $\left|\left(s_{i_{\zeta}}-s_{0}\right) n_{1_{\zeta}}\right|<\omega$ ). The proofs of Corollary 7.3 and Theorem 7.4 are in Appendix D.

Theorem 7.4 shows that $\left\{\mathcal{A}_{\zeta}\right\}$ is weakly asymptotically extractive, i.e., the total bidders' profit is bounded, if and only if proportional selling is used: it is weakly extractive if $[i]$ holds and it is not efficient (and therefore not extractive) if $[i i]$ holds. ${ }^{25}$ If neither $[i]$ nor $[i i]$ hold, then the sequence of auctions is asymptotically efficient but might not be weakly asymptotically extractive. An important special case are auctions in which the number of objects $k_{i}$ cannot be varied, i.e., $k_{i} \equiv k \forall i$.

\footnotetext{
25 This result assumes $0<\lim _{\zeta \rightarrow \infty} s_{i_{\zeta}}<1$. Under an additional assumption that $h(t) \neq 0 \Rightarrow h(t)>\theta$ for some $\theta>0$, which is similar to the Assumption (A1) of Jackson and Kremer (2005, pg. 11), all asymptotic approximations are valid for $0 \leq \lim _{\zeta \rightarrow \infty} s_{i_{\zeta}} \leq 1$.
} 
Corollary 7.5 Let Assumptions 7.1 and 7.2 hold for a sequence of auctions $\left\{\mathcal{A}_{\zeta}\right\}_{\zeta=1,2, \ldots}$ such that $k_{i_{\zeta}}=k_{\zeta}$ for all $i, \zeta$. Suppose that $0<\liminf \operatorname{in}_{\zeta \rightarrow \infty} k_{\zeta} / n_{M_{\zeta}} \leq \lim \sup _{\zeta \rightarrow \infty} k_{\zeta} / n_{1_{\zeta}}<1$. Then $\lim \sup _{\zeta} S\left(\Omega_{\mathbf{k}_{\zeta}}\right)<\infty$ if there exists $\omega$ such that $\left|n_{M_{\zeta}}-n_{1_{\zeta}}\right|<\omega$ and $\lim \sup _{\zeta} S\left(\Omega_{\mathbf{k}_{\zeta}}\right)=\infty$ if $\lim _{\zeta \rightarrow \infty} n_{M_{\zeta}} / n_{1_{\zeta}}>1$.

\section{Appendix B: Proofs and Derivations}

\subsection{Proof of Theorem 3.1.}

Assume that an increasing function $b_{0}$ is a symmetric equilibrium bid function. Hence the bid $b_{0}(x)$ maximizes expected profit for a bidder who observes the signal $x$ when all rivals use $b_{0}$. Consider such a bidder observing signal $x$. Denote by $f_{n-1, k}(y \mid v)$ the conditional probability density function of $Y_{n-1}^{k}$, the $k^{\text {th }}$-highest order statistic out of $n-1$ signals, given $v$ :

$$
f_{n-1, k}(y \mid v)=\frac{(n-1) !}{(k-1) !(n-k-1) !} f(y \mid v) F^{n-k-1}(y \mid v)(1-F(y \mid v))^{k-1} .
$$

By assumption $b_{0}(x)$ is increasing but does not have to be continuous. Define $b_{0}^{-1}(b)=$ $\sup \left\{x: b_{0}(x) \leq b\right\}$, i.e., $b_{0}^{-1}(b)$ is the largest $x$ such that $b_{0}(x) \leq b$. Then $b_{0}^{-1}$ is defined for all $b$ and, for all $x, x=b_{0}^{-1}\left(b_{0}(x)\right)$. Conditional on a realized common value $v$, and numbers of bidders and objects $\left(n_{i}, k_{i}\right)$, expected profit $\Pi_{i}(b \mid v)$ of a bidder that bids $b$ when all rivals use the function $b_{0}$ is:

$$
\Pi_{i}(b \mid v)=\int_{-\infty}^{b_{0}^{-1}(b)}\left(v-b_{0}(y)\right) f_{n_{i}-1, k_{i}}(y \mid v) d y .
$$

Using Matthews (1987), the probability of $n_{i}$ bidders is $\pi_{i} n_{i} / \bar{n}$, where $\bar{n}=\sum_{i=1}^{M} \pi_{i} n_{i}$. Denote by $g(v \mid x)$ the distribution of $v$ given $x$, which by Bayes' Theorem is:

$$
g(v \mid x)=\frac{f(x \mid v) g(v)}{\int_{\underline{v}}^{\bar{v}} f(x \mid t) g(t) d t} .
$$

Ex ante unconditional expected profit for a bidder that observes signal $x$ and bids $b$ (i.e., taking the expectation over $v$ and all pairs $\left.\left(n_{i}, k_{i}\right)\right)$, is

$$
\begin{aligned}
\Pi(b, x) & =\sum_{i=1}^{M} \frac{\pi_{i} n_{i}}{\bar{n}} \int_{\underline{v}}^{\bar{v}} \Pi_{i}(b \mid v) g(v \mid x) d v=\frac{\sum_{i=1}^{M} \pi_{i} n_{i} \int_{\underline{v}}^{\bar{v}} \Pi_{i}(b \mid v) f(x \mid v) g(v) d v}{\bar{n} \int_{\underline{v}}^{\bar{v}} f(x \mid t) g(t) d t} \\
& =\frac{\sum_{i=1}^{M} \pi_{i} n_{i} \int_{\underline{v}}^{\bar{v}}\left(\int_{-\infty}^{b_{0}^{-1}(b)}\left(v-b_{0}(y)\right) f_{n_{i}-1, k_{i}}(y \mid v) d y\right) f(x \mid v) g(v) d v}{\bar{n} \int_{\underline{v}}^{\bar{v}} f(x \mid t) g(t) d t} .
\end{aligned}
$$


Define

$$
v(x, y)=\frac{\sum_{i=1}^{M} \pi_{i} n_{i} \int_{\underline{v}}^{\bar{v}} v f_{n_{i}-1, k_{i}}(y \mid v) f(x \mid v) g(v) d v}{\sum_{i=1}^{M} \pi_{i} n_{i} \int_{\underline{v}}^{\bar{v}} f_{n_{i}-1, k_{i}}(y \mid t) f(x \mid t) g(t) d t},
$$

and denote by $f_{Y}$ the density of the pivotal rival signal:

$$
f_{Y}(y \mid x)=\frac{\sum_{i=1}^{M} \pi_{i} n_{i} \int_{\underline{v}}^{\bar{v}} f_{n_{i}-1, k_{i}}(y \mid v) f(x \mid v) g(v) d v}{\bar{n} \int_{\underline{v}}^{\bar{v}} f(x \mid t) g(t) d t} .
$$

Then, by Fubini's theorem, (10) can be expressed as

$$
\begin{aligned}
\Pi(b, x) & =\frac{\int_{-\infty}^{b_{0}^{-1}(b)}\left(\sum_{i=1}^{M} \pi_{i} n_{i} \int_{\underline{v}}^{\bar{v}} v f_{n_{i}-1, k_{i}}(y \mid v) f(x \mid v) g(v) d v\right) d y}{\bar{n} \int_{\underline{v}}^{\bar{v}} f(x \mid t) g(t) d t}-\int_{-\infty}^{b_{0}^{-1}(b)} b_{0}(y) f_{Y}(y \mid x) d y \\
& =\frac{\int_{-\infty}^{b_{0}^{-1}(b)} v(x, y)\left(\sum_{i=1}^{M} \pi_{i} n_{i} \int_{\underline{v}}^{\bar{v}} f_{n_{i}-1, k_{i}}(y \mid v) f(x \mid v) g(v) d v\right) d y}{\bar{n} \int_{\underline{v}}^{\bar{v}} f(x \mid t) g(t) d t}-\int_{-\infty}^{b_{0}^{-1}(b)} b_{0}(y) f_{Y}(y \mid x) d y \\
& =\int_{-\infty}^{b_{0}^{-1}(b)}\left(v(x, y)-b_{0}(y)\right) f_{Y}(y \mid x) d y
\end{aligned}
$$

where, obviously, $f_{Y}(y \mid x)$ is non-negative. Also note that $v(x, y)$ is increasing in its first argument, and is continuous. To show this rewrite (11) (using equation (3.5) in Milgrom, 1981):

$$
v(x, y)=\frac{\int_{\underline{v}}^{\bar{v}} v f(x \mid v) g_{y}(v) d v}{\int_{\underline{v}}^{\bar{v}} f(x \mid t) g_{y}(t) d t}
$$

where $g_{y}(v)=\sum_{i=1}^{M} \pi_{i} n_{i} f_{n_{i}-1, k_{i}}(y \mid v) g(v) / \bar{n}$. Since $f(\cdot \mid v)$ satisfies $\operatorname{SMLRP}$ (eq. (1)), by Theorem 2.1 of Milgrom (1981), for every nondegenerate prior distribution $G$ with pdf $g$ and every $x_{L}$ and $x_{U}$ in the support of $X_{1}$ such that $x_{L}<x_{U}, G\left(\cdot \mid X=x_{U}\right)$ dominates $G\left(\cdot \mid X=x_{L}\right)$ in the sense of strict first-order stochastic dominance. In particular, for $G_{y}$ with pdf $g_{y}$

$$
x_{L}<x_{U} \Rightarrow v\left(x_{L}, y\right)=E\left[V \mid x_{L}\right]<E\left[V \mid x_{U}\right]=v\left(x_{U}, y\right),
$$

so the claim follows.

In order to show that $b^{*}$, given by (4), is a symmetric equilibrium if it is increasing, first note that setting $x=y$ in (11) yields $b^{*}=v(x, x)$ (and $b^{*}$ is continuous). Suppose $v(x, x)$ is increasing. Then, using (12),

$$
\Pi(b, x)-\Pi\left(b^{*}(x), x\right)=\int_{x}^{b^{*-1}(b)}(v(x, y)-v(y, y)) f_{Y}(y \mid x) d y .
$$

Thus, since $v(x, y)$ is increasing in its first argument, (14) is non-positive for all $b \neq b^{*}(x)$. Hence the bid $b^{*}(x)$ maximizes expected profit for a bidder who observes the signal $x$ when all rivals use $b^{*}$, i.e., $b^{*}$ is a symmetric equilibrium bid function. 
Finally, suppose there exists an increasing symmetric equilibrium bid function $b_{0}, b_{0} \neq b^{*}$, i.e., suppose that there exists $x_{0}$ such that $b_{0}\left(x_{0}\right) \neq v\left(x_{0}, x_{0}\right)$. Consider the case $b_{0}\left(x_{0}\right)<$ $v\left(x_{0}, x_{0}\right)$ (the case $b_{0}\left(x_{0}\right)>v\left(x_{0}, x_{0}\right)$ is treated similarly). If $b_{0}$ is continuous from the right at $x_{0}$, then there exist $x_{1}<x_{2}$ in the neighborhood of $x_{0}$ such that for all $y, x_{1} \leq y \leq x_{2}$, $b_{0}(y)<v\left(x_{1}, y\right)$ and $f_{Y}\left(y \mid x_{1}\right)>0$. Then, using (12),

$$
\Pi\left(b_{0}\left(x_{2}\right), x_{1}\right)-\boldsymbol{\Pi}\left(b_{0}\left(x_{1}\right), x_{1}\right)=\int_{x_{1}}^{x_{2}}\left(v\left(x_{1}, y\right)-b_{0}(y)\right) f_{Y}(y \mid x) d y>0,
$$

so a bidder observing signal $x_{1}$ benefits by deviating from bidding $b_{0}\left(x_{1}\right)$ to bidding $b_{0}\left(x_{2}\right)$. If $b_{0}\left(x_{0}\right)$ is discontinuous from the right at $x_{0}$, then there exists $b_{1}, b_{0}\left(x_{0}\right)<b_{1}<v\left(x_{0}, x_{0}\right)$. Note that by definition $b_{0}^{-1}\left(b_{1}\right)=b_{0}^{-1}\left(b_{0}\left(x_{0}\right)\right)=x_{0}$. A bidder that observes signal $x_{0}$ benefits by bidding $b_{1}$ instead of $b_{0}\left(x_{0}\right)$ in the case of a tie with the pivotal bid (recall that ties are settled randomly): conditional on a pivotal bidder having signal $x_{0}$, the expected value is $v\left(x_{0}, x_{0}\right)$ and the price is $b\left(x_{0}\right)<v\left(x_{0}, x_{0}\right)$.

Therefore, if $b^{*}$ is increasing, it is the unique symmetric equilibrium in increasing strategies.

Remark 8.1 Pesendorfer and Swinkels' (1997) proof that an equilibrium bid function cannot have "flat segments" is a key step in showing that a symmetric equilibrium is increasing. As illustrated below, the "loser's curse" characterization in their Lemma 7 does not extend to auctions with an unknown number of bidders.

Suppose bidders all use nondecreasing bid function $b^{0}$ (which might not be an equilibrium strategy) such that there exists $b \in(\underline{v}, \bar{v})$ and a nondegenerate interval $I=\left\{x \in \Re \mid b^{0}(x)=b\right\}$. Consider bidder 1 observing signal $X_{1}=x \in I$. Let $Y$ be the pivotal rival's signal (i.e., the $k_{i}^{\text {th }}$ highest of $n_{i}-1$ rival signals), and denote by $E\left[V \mid b\right.$ wins, $\left.Y \in I, X_{1}=x\right]$ (resp., $E\left[V \mid b\right.$ loses, $\left.\left.Y \in I, X_{1}=x\right]\right)$ expected value given that bidder 1 wins (loses) by bidding $b$, that the pivotal rival's bid is $b$ (i.e., that $Y \in I$ ), and that he observed $x$. Pesendorfer and Swinkels find that, for known number of bidders,

$$
E\left[V \mid b \text { wins, } Y \in I, X_{1}=x\right]<E\left[V \mid b \text { loses, } Y \in I, X_{1}=x\right] .
$$

The intuition: a bidder is more likely to lose a tie-breaker when tied with more of the known $n-1$ rivals and when more rivals bid above $b$, which in turn is more likely when the objects' value is higher. In contrast, when the number of bidders is unknown, losing a tie-breaker may simply mean that there were more bids submitted, which might overwhelm any inference about $E[V]$ drawn from losing a tie-breaker given a particular number of participating bidders. So, if the number of bidders is unknown, it is possible that

$$
E\left[V \mid b \text { wins, } Y \in I, X_{1}=x\right]>E\left[V \mid b \text { loses, } Y \in I, X_{1}=x\right] \text {. }
$$


For example, consider an auction for a single object, with two or four possible bidders, uniform prior and uniform signal distribution, i.e., let $\mathcal{A}=\left\{\Omega_{\mathbf{k}}, g(\cdot), f(\cdot)\right\}$ with $\Omega_{\mathbf{k}}=$ $\{(2,1,0.6),(4,1,0.4)\}, g(v) \sim U[-1,1.1]$, and $f(x \mid v) \sim U[v-0.5, v+0.5]$.

Let $b^{0}$ be a nondecreasing bid function such that there exists $b \in(-1,1.1)$ with $I=$ $\left\{x \in \Re \mid b^{0}(x)=b\right\}=[0,0.1]$. Suppose that all bidders use $b^{0}$. Then

$\mathrm{E}\left[\mathrm{V} \mid \mathrm{b}\right.$ wins, $\left.\mathrm{Y} \in \mathrm{I}, \mathrm{X}_{1}=0\right] \approx-0.0328>\mathrm{E}\left[\mathrm{V} \mid \mathrm{b}\right.$ loses, $\left.\mathrm{Y} \in \mathrm{I}, \mathrm{X}_{1}=0\right] \approx-0.0347$

To check (15), first note that, given $v$, for 1 object and $n$ bidders, the probability that $j$ rival signals, including the pivotal rival's signal $Y$, are in $I$ is:

$$
\frac{(n-1) !}{j !(n-j-1) !} F(0 \mid v)^{n-j-1}(F(0.1 \mid v)-F(0 \mid v))^{j} .
$$

Thus, given that $j$ rivals are tied with bidder 1 , then (since ties are broken at random) the probability of bidder 1 winning is $1 /[j+1]$ and the probability of bidder 1 losing is $j /[j+1]$. Given $v$, the joint event probabilities are

$$
\begin{aligned}
& \operatorname{Pr}\left[b \text { wins, } Y \in I, X_{1}=0 \mid v\right] \\
& =f(0 \mid v) \sum_{i=1}^{M} \frac{\pi_{i} n_{i}}{\bar{n}} \sum_{j=1}^{n_{i}-1} \frac{1}{j+1} \frac{\left(n_{i}-1\right) !}{j !\left(n_{i}-j-1\right) !} F(0 \mid v)^{n_{i}-j-1}(F(0.1 \mid v)-F(0 \mid v))^{j} \\
& \operatorname{Pr}\left[b \text { loses, } Y \in I, X_{1}=0 \mid v\right] \\
& =f(0 \mid v) \sum_{i=1}^{M} \frac{\pi_{i} n_{i}}{\bar{n}} \sum_{j=1}^{n_{i}-1} \frac{j}{j+1} \frac{\left(n_{i}-1\right) !}{j !\left(n_{i}-j-1\right) !} F(0 \mid v)^{n_{i}-j-1}(F(0.1 \mid v)-F(0 \mid v))^{j}
\end{aligned}
$$

Then

$$
\begin{aligned}
& E\left[V \mid b \text { wins, } Y \in I, X_{1}=0\right]=\frac{\int_{\underline{v}}^{\bar{v}} v \operatorname{Pr}\left[b \text { wins, } Y \in I, X_{1}=0 \mid v\right] g(v) d v}{\int_{\underline{v}}^{\bar{v}} \operatorname{Pr}\left[b \text { wins, } Y \in I, X_{1}=0 \mid t\right] g(t) d t} \\
= & \frac{\sum_{i=1}^{M} \pi_{i} \sum_{j=1}^{n_{i}-1} \frac{n_{i} !}{(j+1) !\left(n_{i}-j-1\right) !} \int_{\underline{v}}^{\bar{v}} v F(0 \mid v)^{n_{i}-j-1}(F(0.1 \mid v)-F(0 \mid v))^{j} f(0 \mid v) g(v) d v}{\sum_{i=1}^{M} \pi_{i} \sum_{j=1}^{n_{i}-1} \frac{n_{i} !}{(j+1) !\left(n_{i}-j-1\right) !} \int_{\underline{v}}^{\bar{v}} F(0 \mid t)^{n_{i}-j-1}(F(0.1 \mid t)-F(0 \mid t))^{j} f(0 \mid t) g(t) d t} ; \\
& E\left[V \mid b \operatorname{loses}, Y \in I, X_{1}=0\right]=\frac{\int_{\underline{v}}^{\bar{v}} v \operatorname{Pr}\left[b \text { loses, } Y \in I, X_{1}=0 \mid v\right] g(v) d v}{\int_{\underline{v}}^{\bar{v}} \operatorname{Pr}\left[b \text { loses, } Y \in I, X_{1}=0 \mid t\right] g(t) d t} \\
= & \frac{\sum_{i=1}^{M} \pi_{i} \sum_{j=1}^{n_{i}-1} \frac{n_{i} ! j}{(j+1) !\left(n_{i}-j-1\right) !} \int_{\underline{v}}^{\bar{v}} v F(0 \mid v)^{n_{i}-j-1}(F(0.1 \mid v)-F(0 \mid v))^{j} f(0 \mid v) g(v) d v}{\sum_{i=1}^{M} \pi_{i} \sum_{j=1}^{n_{i}-1} \frac{n_{i} ! j}{(j+1) !\left(n_{i}-j-1\right) !} \int_{\underline{v}}^{\bar{v}} F(0 \mid t)^{n_{i}-j-1}(F(0.1 \mid t)-F(0 \mid t))^{j} f(0 \mid t) g(t) d t} .
\end{aligned}
$$

Direct numerical calculations of these two equations yield (15). 


\subsection{Derivation of Example 3.2}

Consider an auction for $k=1$ object with $\Omega_{\mathbf{k}}=\left\{\left(2,1, \pi_{1}\right),\left(n_{2}, 1,1-\pi_{1}\right)\right\}$. Let $g(v)$ be uniform on $[0 ; \bar{v}], \bar{v}>1$, and let $f(x \mid v)$ be uniform on $[v-1 / 2 ; v+1 / 2]$. Then $f(x \mid v)=1$ if $|x-v| \leq 1 / 2$ and $f(x \mid v)=0$ if $|x-v|>1 / 2$. Correspondingly, $F(x \mid v)=x-v+1 / 2$ if $|x-v| \leq 1 / 2, F(x \mid v)=0$ if $x<v-1 / 2$ and $F(x \mid v)=1$ if $x>v-1 / 2$. Support of the posterior distribution of $v$ is bounded by $\underline{v_{x}}=\max (0, x-1 / 2), \overline{v_{x}}=\min (\bar{v}, x+1 / 2)$. The support of $X_{1}$ is $[-1 / 2 ; \bar{v}+1 / 2]$. Equation (4) becomes

$$
b^{*}(x)=\frac{\sum_{i=1}^{2} \pi_{i} n_{i}\left(n_{i}-1\right) \int_{\underline{v_{x}}}^{\overline{v_{x}}} v\left(x-v+\frac{1}{2}\right)^{n_{i}-2} d v}{\sum_{i=1}^{2} \pi_{i} n_{i}\left(n_{i}-1\right) \int_{\underline{v_{x}}}^{\overline{v_{x}}}\left(x-v+\frac{1}{2}\right)^{n_{i}-2} d v} .
$$

Changing variables $t=x-v+1 / 2$ yields $d v=-d t, v=x-t+1 / 2$, so

$$
b^{*}(x)=\frac{\sum_{i=1}^{2} \pi_{i} n_{i}\left(n_{i}-1\right) \int_{x-\underline{v_{x}}+1 / 2}^{x-\overline{v_{x}}+1 / 2}\left(x-t+\frac{1}{2}\right) t^{n_{i}-2} d t}{\sum_{i=1}^{2} \pi_{i} n_{i}\left(n_{i}-1\right) \int_{x-\underline{v_{x}}+1 / 2}^{x-\bar{x}+1 / 2} t^{n_{i}-2} d t} .
$$

Consider $x$ such that $x<\bar{v}-1 / 2$. In that case $x-\underline{v_{x}}+1 / 2=\min (x+1 / 2,1), x-\overline{v_{x}}+1 / 2=0$, and (16) becomes

$$
\begin{aligned}
b^{*}(x) & =\frac{\sum_{i=1}^{2} \pi_{i} n_{i}\left(n_{i}-1\right) \int_{\min (x+1 / 2,1)}^{0}\left(x-t+\frac{1}{2}\right) t^{n_{i}-2} d t}{\sum_{i=1}^{2} \pi_{i} n_{i}\left(n_{i}-1\right) \int_{\min (x+1 / 2,1)}^{0} t^{n_{i}-2} d t} \\
& =\frac{\sum_{i=1}^{2} \pi_{i} n_{i}\left(n_{i}-1\right)\left(\left(x+\frac{1}{2}\right) \frac{1}{n_{i}-1}\left(\min \left(x+\frac{1}{2}, 1\right)\right)^{n_{i}-1}-\frac{1}{n_{i}}\left(\min \left(x+\frac{1}{2}, 1\right)\right)^{n_{i}}\right)}{\sum_{i=1}^{2} \pi_{i} n_{i}\left(n_{i}-1\right) \frac{1}{n_{i}-1}\left(\min \left(x+\frac{1}{2}, 1\right)\right)^{n_{i}-1}} \\
& =x+\frac{1}{2}-\frac{\sum_{i=1}^{2} \pi_{i}\left(n_{i}-1\right)\left(\min \left(x+\frac{1}{2}, 1\right)\right)^{n_{i}}}{\sum_{i=1}^{2} \pi_{i} n_{i}\left(\min \left(x+\frac{1}{2}, 1\right)\right)^{n_{i}-1}} .
\end{aligned}
$$

Consider $x<1 / 2$. Using $n_{1}=2$,

$$
\begin{aligned}
b^{*}(x) & =x+\frac{1}{2}-\frac{\pi_{1}\left(x+\frac{1}{2}\right)^{2}+\left(1-\pi_{1}\right)\left(n_{2}-1\right)\left(x+\frac{1}{2}\right)^{n_{2}}}{\pi_{1} 2\left(x+\frac{1}{2}\right)+\left(1-\pi_{1}\right) n_{2}\left(x+\frac{1}{2}\right)^{n_{2}-1}} \\
& =x+\frac{1}{2}-\frac{\pi_{1}\left(x+\frac{1}{2}\right)+\left(1-\pi_{1}\right)\left(n_{2}-1\right)\left(x+\frac{1}{2}\right)^{n_{2}-1}}{2 \pi_{1}+\left(1-\pi_{1}\right) n_{2}\left(x+\frac{1}{2}\right)^{n_{2}-2}} .
\end{aligned}
$$

\subsection{Proof of Lemma 4.1.}

Recall that the (untruncated) beta distribution with parameters $n-k$ and $k$ is defined by its pdf

$$
\beta(y ; n-k, k)=\frac{y^{n-k-1}(1-y)^{k-1}}{\int_{0}^{1} t^{n-k-1}(1-t)^{k-1} d t},
$$


and that it has expected value $1-k / n$ and variance $k(n-k) /\left[n^{2}(n+1)\right]<1 / n$. Also recall that $\beta(y ; n-k, k)$ has a unique maximum at $1-(k-1) /(n-2)$ for $n>2$.

Let $0 \leq \underline{y}<\bar{y} \leq 1$ and define the truncated beta distribution with parameters $n-k$ and $k$ on $[\underline{y}, \bar{y}]$ by its pdf

$$
\beta^{T}(y ; n-k, k)=\frac{y^{n-k-1}(1-y)^{k-1}}{\int_{\underline{y}}^{\bar{y}} t^{n-k-1}(1-t)^{k-1} d t} .
$$

Denote its expected value by $E_{\beta^{T}}$ and its variance by $\operatorname{Var}_{\beta^{T}}$. To avoid overly convoluted notation, the dependence of $\beta^{T}, E_{\beta^{T}}$ and $\operatorname{Var}_{\beta^{T}}$ on $\underline{y}, \bar{y}$ is submersed. Note that $\operatorname{Var}_{\beta^{T}}<1 / n$ since the variance of the truncated beta distribution (18) is no higher than the variance of the corresponding (untruncated) beta distribution (17). Also, by properties of beta distributions, there exists a constant $\theta>0$ such that $\left|E_{\beta^{T}}-y_{n k}\right|<\theta / n$, where

$$
y_{n k}= \begin{cases}\bar{y}, & k / n<1-\bar{y}, \\ 1-k / n, & 1-\bar{y} \leq k / n \leq 1-\underline{y}, \\ \underline{y}, & 1-\underline{y}<k / n .\end{cases}
$$

Concentration properties of truncated beta distributions (similar to Ito's Lemma), described in Lemma 9.1 in Appendix C, play an important role in the proof of Lemma 4.1.

\section{Proof of Lemma 4.1.}

Consider a bidder in auction $\mathcal{A}_{u}$, and, for the remainder of the proof, fix arbitrarily the signal $x \in \operatorname{supp}\left(X_{1}\right)$ he observes.

Define $\gamma_{x}(t)$ as the solution to the equation

$$
F\left(x \mid \gamma_{x}(t)\right)=t
$$

Let $\left[\underline{v_{x}}, \overline{v_{x}}\right]$ be the support of the posterior distribution $g(v \mid x)=f(x \mid v) g(v) / \int_{\underline{v}}^{\bar{v}} f(x \mid t) g(t) d t$. Denote

$$
\underline{y}=F\left(x \mid \overline{v_{x}}\right), \quad \bar{y}=F\left(x \mid \underline{v_{x}}\right) .
$$

Note that $f(x \mid v)$ satisfies SMLRP, so $f(x \mid v)$ first-order stochastically dominates $f\left(x \mid v^{\prime}\right)$ for all $v>v^{\prime}$, i.e., $F(x \mid v)<F\left(x \mid v^{\prime}\right) \forall x, v, v^{\prime}$ such that $v>v^{\prime}$. Therefore, $\gamma_{x}(t)$ is strictly decreasing on $(\underline{y}, \bar{y})$. Also note that, by the Implicit Function Theorem, $\gamma_{x}$ is thrice differentiable. Denote by $\gamma_{x}^{\prime}$ the derivative of $\gamma_{x}$. Note that $\gamma_{x}^{\prime}(y)=1 / \frac{\partial F\left(x \mid \gamma_{x}(y)\right)}{\partial v}<0$ exists by assumption.

Using (9) and substituting $\gamma_{x}(y)$ for $v$ (and using $d v=\gamma_{x}^{\prime}(y) d y$ ) in equation (2) yields

$$
\begin{aligned}
b_{n_{u} k_{u}}(x) & =\frac{\int_{\underline{v_{x}}}^{\overline{v_{x}}} v f_{n_{u}-1, k_{u}}(x \mid v) f(x \mid v) g(v) d v}{\int_{\underline{v_{x}}}^{\overline{v_{x}}} f_{n_{u}-1, k_{u}}(x \mid t) f(x \mid t) g(t) d t} \\
& =\frac{\int_{\bar{y}}^{\underline{y}} \gamma_{x}(y) f\left(x \mid \gamma_{x}(y)\right) y^{n_{u}-k_{u}-1}(1-y)^{k_{u}-1} f\left(x \mid \gamma_{x}(y)\right) g\left(\gamma_{x}(y)\right) \gamma_{x}^{\prime}(y) d y}{\int_{\bar{y}}^{\frac{y}{y}} f\left(x \mid \gamma_{x}(t)\right) t^{n_{u}-k_{u}-1}(1-t)^{k_{u}-1} f\left(x \mid \gamma_{x}(t)\right) g\left(\gamma_{x}(t)\right) \gamma_{x}^{\prime}(t) d t} .
\end{aligned}
$$


In turn, this expression can be rewritten via a truncated beta distribution (18). Then

$$
b_{n_{u} k_{u}}(x)=\frac{\int_{\underline{y}}^{\bar{y}} \gamma_{x}(y) f^{2}\left(x \mid \gamma_{x}(y)\right) g\left(\gamma_{x}(y)\right) \gamma_{x}^{\prime}(y) \beta^{T}\left(y ; n_{u}-k_{u}, k_{u}\right) d y}{\int_{\underline{y}}^{\bar{y}} f^{2}\left(x \mid \gamma_{x}(t)\right) g\left(\gamma_{x}(t)\right) \gamma_{x}^{\prime}(t) \beta^{T}\left(t ; n_{u}-k_{u}, k_{u}\right) d t} .
$$

Applying Lemma 9.1 to both numerator and denominator of (20) yields ${ }^{26}$

$$
b_{n_{u} k_{u}}(x)=\frac{\gamma_{x}\left(y_{n_{u} k_{u}}\right) f^{2}\left(x \mid \gamma_{x}\left(y_{n_{u} k_{u}}\right)\right) g\left(\gamma_{x}\left(y_{n_{u} k_{u}}\right)\right) \gamma_{x}^{\prime}\left(y_{n_{u} k_{u}}\right)+O\left(\frac{1}{n_{u}}\right)}{f^{2}\left(x \mid \gamma_{x}\left(y_{n_{u} k_{u}}\right)\right) g\left(\gamma_{x}\left(y_{n_{u} k_{u}}\right)\right) \gamma_{x}^{\prime}\left(y_{n_{u} k_{u}}\right)+O\left(\frac{1}{n_{u}}\right)}=\gamma_{x}\left(y_{n_{u} k_{u}}\right)+O\left(\frac{1}{n_{u}}\right) .
$$

Defining $\kappa$ via

$$
\kappa(x, t)= \begin{cases}\gamma_{x}(\bar{y}), & t<1-\bar{y}, \\ \gamma_{x}(1-t), & 1-\bar{y} \leq t \leq 1-\underline{y}, \\ \gamma_{x}(\underline{y}), & t>1-\underline{y}\end{cases}
$$

completes the proof.

Remark 8.2 Equation (21) states only pointwise convergence. However, by Lemma 9.1, the bounds in both numerator and denominator are continuous in $x$, and thus are bounded for any compact set. Therefore, Lemma 4.1 can be strengthened as follows: for any compact set $\mathcal{D}$ in the support of $X_{1}$ and any $\varepsilon>0$ there exists $A$ and $n^{*}$ such that $\left|b_{n_{u} k_{u}}(x)-\kappa\left(x, s_{u}\right)\right|<A / n_{u}$ if $\varepsilon<k_{u} / n_{u}<1-\varepsilon, n_{u}>n^{*}$, and $x \in \mathcal{D}$.

Proof. Denote $q(x)=f^{2}\left(x \mid \gamma_{x}\left(y_{n_{u} k_{u}}\right)\right) g\left(\gamma_{x}\left(y_{n_{u} k_{u}}\right)\right) \gamma_{x}^{\prime}\left(y_{n_{u} k_{u}}\right)$, and note that $q(x)<0$ for all $x \in \mathcal{D}$, because $\gamma_{x}^{\prime}(y)<0$ as shown in the proof of Lemma 4.1. By Lemma 9.1, (20) can be expressed as

$$
b_{n_{u} k_{u}}(x)=\frac{\gamma_{x}\left(y_{n_{u} k_{u}}\right) q(x)+a_{1}(x) / n_{u}}{q(x)+a_{2}(x) / n_{u}}=\kappa\left(x, s_{u}\right) \frac{1+\frac{a_{1}(x)}{q(x)} / n_{u}}{1+\frac{a_{2}(x)}{q(x)} / n_{u}} .
$$

where $a_{1}(x)$ and $a_{2}(x)$ are continuous. Let $A_{1}=\max _{x \in \mathcal{D}}\left|\frac{a_{1}(x)}{q(x)}\right|, A_{2}=\max _{x \in \mathcal{D}}\left|\frac{a_{2}(x)}{q(x)}\right|$. Since $\mathcal{D}$ is compact, such $A_{1}$ and $A_{2}$ exist. Then, for $n_{u}>\frac{1}{2 A_{2}}, 1-A_{1} / n_{u} \leq 1+\frac{a_{1}(x)}{q(x)} / n_{u} \leq 1+A_{1} / n_{u}$ and $1-A_{2} / n_{u} \leq \frac{1}{1+A_{2} / n_{u}} \leq \frac{1}{1+\frac{a_{2}(x)}{q(x)} / n_{u}} \leq \frac{1}{1-A_{2} / n_{u}} \leq 1+2 A_{2} / n_{u}$. Therefore,

$$
\left(1-A_{1} / n_{u}\right)\left(1-A_{2} / n_{u}\right) \leq \frac{1+\frac{a_{1}(x)}{q(x)} / n_{u}}{1+\frac{a_{2}(x)}{q(x)} / n_{u}} \leq\left(1+A_{1} / n_{u}\right)\left(1+2 A_{2} / n_{u}\right) .
$$

\footnotetext{
26 Using $\Upsilon(x, y)=\gamma_{x}(y) f^{2}\left(x \mid \gamma_{x}(y)\right) g\left(\gamma_{x}(y)\right) \gamma_{x}^{\prime}(y)$ for the numerator, and, separately, $\Upsilon(x, y)=$ $f^{2}\left(x \mid \gamma_{x}(y)\right) g\left(\gamma_{x}(y)\right) \gamma_{x}^{\prime}(y)$ for the denominator. Note that the last equality in (21) holds because the function of $y_{n_{u} k_{u}}$ in the denominator is negative and, for $q \neq 0,[p+O(1 / n)] /[q+O(1 / n)]=p / q+O(1 / n)$.
} 
Finally,

$$
\left|b_{n_{u} k_{u}}(x)-\kappa\left(x, s_{u}\right)\right|=\left|\kappa\left(x, s_{u}\right)\right| \frac{1+\frac{a_{1}(x)}{q(x)} / n_{u}}{1+\frac{a_{2}(x)}{q(x)} / n_{u}}-1|\leq| \kappa\left(x, s_{u}\right) \mid\left(\frac{A_{1}+2 A_{2}+2 A_{1} A_{2} / n_{u}}{n_{u}}\right) .
$$

Setting $A=\left(A_{1}+2 A_{2}+1\right) \max _{s \in[\varepsilon, 1-\varepsilon], x \in \mathcal{D}}|\kappa(x, s)|$ and $n^{*}=\max \left(\frac{1}{2 A_{2}}, 2 A_{1} A_{2}\right)$ completes the proof.

\subsection{Proof of Theorem 4.2.}

Preparation for the proof considers the properties of simpler sequences of auctions. First consider auctions in which both the number of bidders $n$ and the number of objects $k$ are known. Specifically, consider a vector of sequences of auctions $\left(\left\{A_{r}^{[i]}\right\}_{r=1,2, \ldots}\right)_{i=1, \ldots, M}$ constructed based on $\left\{\mathcal{A}_{\zeta}\right\}$ by setting $\Omega_{\mathbf{k}_{r}}^{[i]}=\left(n_{r}^{[i]}, k_{r}^{[i]}, 1\right)$, where $r=\zeta \Rightarrow\left(n_{r}^{[i]}, k_{r}^{[i]}\right)=\left(n_{i_{\zeta}}, k_{i_{\zeta}}\right) \forall i=$ $1, \ldots, M$. That is, along the $i^{\text {th }}$ sequence, the number of bidders and the number of objects are set nonstochastically at levels matching the $i^{\text {th }}$ element of $\Omega_{\mathbf{k}_{\zeta}}$. Let $P_{r}^{[i]}$ denote the price in $A_{r}^{[i]}$. Then each sequence $\left\{A_{r}^{[i]}\right\}$ satisfies the assumptions of Theorem 1 in Pesendorfer and Swinkels (1997), ${ }^{27}$ so each sequence aggregates information:

$$
\forall i=1, \ldots, M, \forall \varepsilon^{[i]}>0, \quad \lim _{r \rightarrow \infty} \operatorname{Pr}\left[\left|P_{r}^{[i]}-V\right|>\varepsilon^{[i]}\right]=0 .
$$

Next consider a counterfactual sequence of auctions $\left\{A_{r}\right\}_{r=1,2, \ldots}$ with $\{r=\zeta\} \Rightarrow$ $\left\{\Omega_{\mathbf{k}_{r}}=\Omega_{\mathbf{k}_{\zeta}}\right\}$, however with the assumption that the seller is somehow able to reveal to the bidders the number of realized bidders and number of objects being sold prior to bid submission. ${ }^{28}$ Thus, in auction $A_{r}$, when (with probability $\pi_{i}$ ) there are $k_{i_{r}}$ objects sold to $n_{i_{r}}$ bidders, each bidder learns these numbers prior to bid submission and therefore uses bid function $b_{n_{i_{r}} k_{i_{r}}}$. The price in $A_{r}$ is then $P_{R}^{r}$, which combines the $P_{r}^{[i]}$ with probabilities $\pi_{i}{ }^{29}$ Hence, using (22),

$$
\forall \varepsilon>0, \quad \lim _{r \rightarrow \infty} \operatorname{Pr}\left[\left|P_{R}^{r}-V\right|>\varepsilon\right]=0 .
$$

\footnotetext{
${ }^{27}$ Technically, they assume signals have compact support, which we do not. This issue is dealt with carefully below, in reaching (27), and the same treatment can be applied for the next two equations.

${ }^{28}$ Such auctions are counterfactual in the sense that they cannot be described within our model of stochastic competition.

${ }^{29}$ Harstad et al. (1990) point out that this auction could be attained via a contingent-bid auction, in which bidders submit a vector of bids $\left(b^{(1)}, \ldots, b^{(M)}\right)$, with the seller committed to a policy of using the $i^{\text {th }}$ element of each vector corresponding to the number of bids submitted.
} 
Finally consider the original sequence of auctions $\left\{\mathcal{A}_{\zeta}\right\}$. Let $Z_{\zeta}$ denote the price-setting signal in $A_{\zeta}$; this is with probability $\pi_{i}$ the $\left(k_{i_{\zeta}}+1\right)^{\text {st }}$-order statistic out of $n_{i_{\zeta}}$ signals, $i=1, \ldots, M$. This stochastic specification of the price-setting signal, $Z$, is identical, element by element, in the sequences $\left\{A_{r}\right\}$ and $\left\{\mathcal{A}_{\zeta}\right\}$, since $\Omega_{\mathbf{k}_{r}}=\Omega_{\mathbf{k}_{\zeta}}$.

Several aspects relating to the price come together far enough along the sequence $\left\{\mathcal{A}_{\zeta}\right\}$. By proportional selling, for any $\delta_{1}>0$, there exists $\zeta_{1}<\infty$ such that

$$
\zeta>\zeta_{1} \Rightarrow\left|s_{i_{\zeta}}-s\right|<\delta_{1}, \forall i=1, \ldots, M
$$

Let $\mathcal{D}$ be an arbitrary compact subset of the support of $X_{1}$. Since $n_{i_{\zeta}} \rightarrow \infty$ as $\zeta \rightarrow \infty$, by Lemma 4.1, Remark 8.2, and by continuity of $\kappa(x, s)$ and (24), for any $\delta_{2}>0$, there exists $\zeta_{2}$ such that

$$
\left\{\zeta>\zeta_{2}, x \in \mathcal{D}\right\} \Rightarrow\left|b_{n_{i_{\zeta}} k_{i_{\zeta}}}(x)-\kappa(x, s)\right|<\delta_{2}, \forall i=1, \ldots, M .
$$

By $(4), b_{\zeta}^{*}$ is a weighted average of $b_{n_{i_{\zeta}} k_{i_{\zeta}}}, i=1, \ldots, M$. Therefore, using $(25)$, for any $\delta_{3}>0$, there exists $\zeta_{3}$ such that

$$
\left\{\zeta>\zeta_{3}, x \in \mathcal{D}\right\} \Rightarrow\left|b_{n_{i_{\zeta}} k_{i_{\zeta}}}(x)-b_{\zeta}^{*}(x)\right|<\delta_{3}, \forall i=1, \ldots, M .
$$

These preliminaries allow the argument that $\left\{\mathcal{A}_{\zeta}\right\}$ aggregates information to be built, beginning by letting $\varepsilon>0, \delta>0$ be arbitrary. We claim that $P^{\zeta}$ is within $\varepsilon$ of $V$ with probability at least $1-\delta$, for large enough $\zeta$.

To show this claim, let $\mathcal{V}$ be a compact subset of the support of $V$ satisfying $\operatorname{Pr}[v \in \mathcal{V}]>$ $1-\delta / 8$. Specify the heretofore arbitrary $\mathcal{D}$ to be a large enough compact set so that

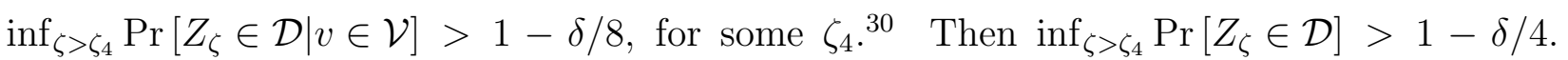
This allows combining (25) and (26) to imply that there exists $\zeta_{5} \geq \zeta_{4}$ so that

$$
\left\{r=\zeta>\zeta_{5}\right\} \Rightarrow \operatorname{Pr}\left[\left|P_{R}^{r}-P^{\zeta}\right|>\frac{\varepsilon}{2} \mid Z_{\zeta} \in \mathcal{D}\right]<\frac{\delta}{4}
$$

and, therefore,

$$
\left\{r=\zeta>\zeta_{5}\right\} \Rightarrow \operatorname{Pr}\left[\left|P_{R}^{r}-P^{\zeta}\right|>\frac{\varepsilon}{2}\right]<\frac{\delta}{2} .
$$

From (23), there exists $\zeta_{6} \geq \zeta_{5}$ so that

$$
\left\{r=\zeta>\zeta_{6}\right\} \Rightarrow \operatorname{Pr}\left[\left|P_{R}^{r}-V\right|>\frac{\varepsilon}{2}\right]<\frac{\delta}{2} .
$$

\footnotetext{
${ }^{30}$ It is possible to make $\mathcal{D}$ large enough to satisfy $\operatorname{Pr}\left[Z_{\zeta} \in \mathcal{D} \mid v \in \mathcal{V}\right]>1-\delta / 8$ for some $\zeta$, call it $\zeta_{4}$, by compactness of $\mathcal{V}$, continuity of $f$, and the law of large numbers. This $\zeta_{4}$ suffices to establish the infimum, as the conditional distribution of $Z_{\zeta}$ narrows as $\zeta$ increases. Note that if the support of $X_{1}$ is compact, then setting $\mathcal{D}$ equal to the support of $X_{1}$ and $\zeta_{4}=1$ suffices.
} 
Finally, the last two developments yield, for all $r=\zeta>\zeta_{6}$ :

$$
\operatorname{Pr}\left[\left|P^{\zeta}-V\right|>\varepsilon\right] \leq \operatorname{Pr}\left[\left|P^{\zeta}-P_{R}^{r}\right|>\frac{\varepsilon}{2}\right]+\operatorname{Pr}\left[\left|P_{R}^{r}-V\right|>\frac{\varepsilon}{2}\right]<\delta,
$$

which completes the proof as $\varepsilon$ and $\delta$ were arbitrary.

\subsection{Proof of Theorem 4.4.}

Denote $v_{U}=\kappa\left(x_{0}, s_{U}\right), v_{L}=\kappa\left(x_{0}, s_{L}\right)$. Denote by $b_{\zeta^{\prime}}^{*}$ the bid function (eq. (4)) that corresponds to $\mathcal{A}_{\zeta^{\prime}}$.

Fix $\varepsilon_{0}=\min \left\{\left(v_{L}-\underline{v}\right) / 2,\left(v_{U}-v_{L}\right) / 4,\left(\bar{v}-v_{U}\right) / 2\right\}>0$. Since $b_{\zeta^{\prime}}^{*}$ is increasing and continuous, there exists a separating sequence $\left\{x_{\zeta^{\prime}}^{*}\right\}_{\zeta^{\prime}=1,2, \ldots}$ corresponding to $\left\{\mathcal{A}_{\zeta^{\prime}}\right\}$ such that $\left|b_{\zeta^{\prime}}^{*}(x)-v_{L}\right| \leq \varepsilon_{0} \Rightarrow x<x_{\zeta^{\prime}}^{*}$ and $\left|b_{\zeta^{\prime}}^{*}(x)-v_{U}\right| \leq \varepsilon_{0} \Rightarrow x>x_{\zeta^{\prime}}^{*}\left(x_{\zeta^{\prime}}^{*}\right.$ is not uniquely determined by this requirement). From a real-valued sequence $\left\{x_{\zeta^{\prime}}^{*}\right\}$, it is always possible to choose an infinite subsequence $\left\{x_{\eta}^{*}\right\}_{\eta=1,2, \ldots}$ which is either nondecreasing or nonincreasing. Suppose $\left\{x_{\eta}^{*}\right\}$ is nondecreasing (the argument for a nonincreasing subsequence is parallel). Denote the corresponding subsequence of auctions by $\left\{\mathcal{A}_{\eta}\right\}$.

Suppose, contrary to the theorem, that the sequence $\left\{\mathcal{A}_{\zeta}\right\}$ aggregates information. Then the subsequence $\left\{\mathcal{A}_{\eta}\right\}$ also aggregates information. For the auction $\mathcal{A}_{\eta}$, let $Z_{\eta}$ be the pricesetting signal and $P^{\eta}$ the auction price. So $P^{\eta}=b_{\eta}^{*}\left(Z_{\eta}\right)$.

Define $\tau(v, s)$ implicitly by $1-F(\tau(v, s) \mid v)=s$, that is, $\tau(v, s)$ is the signal such that a single bidder has a probability $s$ of getting a higher signal, given $v$. Thus,

$$
\lim _{\eta \rightarrow \infty} E\left[Z_{\eta} \mid V=v, n_{i_{\eta}}, k_{i_{\eta}}\right]=\tau\left(v, s_{i}\right), i=L, U
$$

Note that $x_{0}=\tau\left(v_{L}, s_{L}\right)=\tau\left(v_{U}, s_{U}\right)$ by theorem assumption. The order-statistics version of the law of large numbers (see, e.g., David, 1981, or the similar usage for $s=0$ in Wilson, 1977, pg. 515) states that for any $\theta>0$,

$$
\{\underline{v}<v<\bar{v}\} \Rightarrow \lim _{\eta \rightarrow \infty} \operatorname{Pr}\left[\left|Z_{\eta}-\tau\left(v, s_{i}\right)\right| \leq \theta \mid V=v, n_{i_{\eta}}, k_{i_{\eta}}\right]=1, i=L, U,
$$

that is, the distribution of the order statistic collapses to its limit expectation.

Two cases need to be treated, according to whether the nondecreasing sequence $\left\{x_{\eta}^{*}\right\}$ crosses $x_{0}$.

CAsE 1: Suppose there exists $\eta_{0}$ such that $x_{\eta_{0}}^{*}>x_{0}$ (and thus, $x_{\eta}^{*}>x_{0}$ for all $\eta>\eta_{0}$ ). For this case, fix $\lambda=\left(x_{\eta_{0}}^{*}-x_{0}\right) / 2>0$. Set

$$
\varepsilon=\min \left\{\frac{\kappa\left(x_{0}+\lambda, s_{U}\right)-v_{U}}{2}, \frac{v_{U}-\kappa\left(x_{0}-\lambda, s_{U}\right)}{2}, \varepsilon_{0}\right\}
$$


by SMLRP, $\varepsilon>0$. As $g$ is everywhere positive, $\delta_{U}=\operatorname{Pr}\left[\left|V-v_{u}\right| \leq \varepsilon\right]>0$.

For $\left\{\mathcal{A}_{\eta}\right\}$ to aggregate information implies

$$
\lim _{\eta \rightarrow \infty} \operatorname{Pr}\left[\left|P^{\eta}-v_{U}\right|<\varepsilon|| V-v_{U} \mid \leq \varepsilon\right]=1
$$

By construction of $\left\{x_{\eta}^{*}\right\}$, it follows that

$$
\lim _{\eta \rightarrow \infty} \operatorname{Pr}\left[Z_{\eta}<x_{\eta}^{*}|| V-v_{U} \mid \leq \varepsilon\right]=0 .
$$

Thus, if $\left\{\mathcal{A}_{\eta}\right\}$ aggregates information, then for any $\delta>0$ there exists $\eta_{\delta}$ so that

$$
\left\{\eta>\eta_{\delta}\right\} \Rightarrow \operatorname{Pr}\left[\left\{Z_{\eta}<x_{\eta}^{*}\right\} \cap\left\{\left|V-v_{U}\right| \leq \varepsilon\right\}\right]<\delta .
$$

Using (28), there exists $\eta_{\lambda} \geq \eta_{0}$ such that

$$
\left\{\eta>\eta_{\lambda}\right\} \Rightarrow \operatorname{Pr}\left[\left|Z_{\eta}-\tau\left(v_{U}, s_{U}\right)\right| \leq \lambda|| V-v_{U} \mid \leq \varepsilon, n_{U_{\eta}}, k_{U_{\eta}}\right]>\frac{1}{2} .
$$

Combining, from (30), and noting that $x_{0}=\tau\left(v_{U}, s_{U}\right)$,

$$
\left\{\eta>\eta_{\lambda}\right\} \Rightarrow \operatorname{Pr}\left[\left\{Z_{\eta}<x_{\eta}^{*}\right\} \cap\left\{\left|V-v_{U}\right| \leq \varepsilon\right\}\right]>\frac{1}{2} \pi_{U} \delta_{U}
$$

contradicting (29).

CAse 2: Suppose $x_{\eta}^{*} \leq x_{0}$ for all $\eta$. By continuity of $\kappa(x, s)$ in $x$ there exists $x^{\prime}>x_{0}$, such that $\kappa\left(x^{\prime}, s_{L}\right)-v_{L}<\varepsilon_{0}$. For this case, fix $\lambda=\left(x^{\prime}-x_{0}\right) / 2>0$ and denote $v_{L}^{\prime}=\kappa\left(x^{\prime}, s_{L}\right)$. Set

$$
\varepsilon=\min \left\{\frac{\kappa\left(x^{\prime}+\lambda, s_{L}\right)-v_{L}^{\prime}}{2}, \frac{v_{L}^{\prime}-\kappa\left(x^{\prime}-\lambda, s_{L}\right)}{2}, \varepsilon_{0}+v_{L}-v_{L}^{\prime}\right\}>0 .
$$

As before, $\delta_{L}=\operatorname{Pr}\left[\left|V-v_{L}^{\prime}\right| \leq \varepsilon\right]>0$.

The development of a contradiction parallels case 1: for $\left\{\mathcal{A}_{\eta}\right\}$ to aggregate information, for any $\delta>0$ there exists $\eta_{\delta}$ so that

$$
\left\{\eta>\eta_{\delta}\right\} \Rightarrow \operatorname{Pr}\left[\left\{Z_{\eta}>x_{\eta}^{*}\right\} \cap\left\{\left|V-v_{L}^{\prime}\right| \leq \varepsilon\right\}\right]<\delta ;
$$

there exists $\eta_{\lambda}$ such that

$$
\left\{\eta>\eta_{\lambda}\right\} \Rightarrow \operatorname{Pr}\left[\left|Z_{\eta}-\tau\left(v_{L}^{\prime}, s_{L}\right)\right| \leq \lambda|| V-v_{L}^{\prime} \mid \leq \varepsilon, n_{L_{\eta}}, k_{L_{\eta}}\right]>\frac{1}{2}
$$

SO

$$
\left\{\eta>\eta_{\lambda}\right\} \Rightarrow \operatorname{Pr}\left[\left\{Z_{\eta}>x_{\eta}^{*}\right\} \cap\left\{\left|V-v_{L}^{\prime}\right| \leq \varepsilon\right\}\right]>\frac{1}{2} \pi_{L} \delta_{L},
$$

a contradiction that completes the proof. 


\section{Appendix C: A Technical Lemma}

The following lemma plays a role in the proof of Lemma 4.1 and Theorem 7.4.

Lemma 9.1 Let I be an interval of reals and let $\underline{y}(x)$ and $\bar{y}(x), 0 \leq \underline{y}(x)<\bar{y}(x) \leq 1$ be continuous on $I$. Let $\Upsilon(x, y)$ be a real-valued function defined on $\{(x, y) \mid x \in I, y(x) \leq$ $y \leq \bar{y}(x), 0<y<1\}$ such that $\partial^{2} \Upsilon(x, y) / \partial y^{2}$ exists and is continuous, and such that $\int_{\underline{y}(x)}^{\bar{y}(x)}|\Upsilon(x, y)| d y$ exists for every $x \in I$. Let $\varepsilon>0$. Then there exists a continuous function $\bar{C}(\cdot)$ on I such that

$$
\left|\int_{\underline{y}(x)}^{\bar{y}(x)} \Upsilon(x, y) \beta^{T}(y ; n-k, k) d y-\Upsilon\left(x, y_{n k}\right)\right| \leq \frac{1}{n} C(x)
$$

for any $x \in I$ and any $n, k$ such that $2 \varepsilon<k / n<1-2 \varepsilon$.

Proof. Consider the case $\underline{y}(x)>0, \bar{y}(x)=1 .^{31} \quad$ (Note that $\beta^{T}(\cdot)$ in (31) depends upon $\underline{y}(x), \bar{y}(x)$ and thereby is a function of $x$.) Define $\varepsilon_{1}(x)=\frac{1}{8} \min (\varepsilon, 1-\underline{y}(x))$. Note that $\varepsilon_{1}(x)$ is positive and continuous in $x$. Rewrite the left-hand side of (31) as

$$
\begin{aligned}
& \left|\int_{\underline{y}(x)}^{1} \Upsilon(x, y) \beta^{T}(y ; n-k, k) d y-\Upsilon\left(E_{\beta^{T}}\right)\right| \\
& =\left|\int_{\underline{y}(x)}^{1-\varepsilon_{1}(x)} \Upsilon(x, y) \beta^{T}(y ; n-k, k) d y+\int_{1-\varepsilon_{1}(x)}^{1} \Upsilon(x, y) \beta^{T}(y ; n-k, k) d y-\Upsilon\left(x, E_{\beta^{T}}\right)\right| \\
& \leq\left|\int_{1-\varepsilon_{1}(x)}^{1} \Upsilon(x, y) \beta^{T}(y ; n-k, k) d y\right|+\left|\int_{\underline{y}(x)}^{1-\varepsilon_{1}(x)} \Upsilon(x, y) \beta^{T}(y ; n-k, k) d y-\Upsilon\left(x, E_{\beta^{T}}\right)\right| .
\end{aligned}
$$

We will show that both of the terms in (32) are of the order $\operatorname{Var}_{\beta^{T}}$, which in turn is less than $1 / n$. The last step is to show that substituting $y_{n k}$ for $E_{\beta^{T}}$ preserves the order $1 / n$. Consider the first term of (32): Since $k / n>2 \varepsilon, E_{\beta^{T}}<1-4 \varepsilon_{1}(x)$. By Chebyshev's inequality,

$$
\int_{1-2 \varepsilon_{1}(x)}^{1} \beta^{T}(y ; n-k, k) d y \leq \frac{\operatorname{Var}_{\beta^{T}}}{4 \varepsilon_{1}^{2}(x)} .
$$

Let $A_{1}(x)=\beta^{T}\left(1-\varepsilon_{1}(x) ; n-k, k\right)$. Note that $\beta^{T}(y ; n-k, k)$ is decreasing in $y$ for $1-2 \varepsilon_{1}(x)<$ $y<1 .{ }^{32}$ Then

$$
\begin{aligned}
\frac{\operatorname{Var}_{\beta^{T}}}{4 \varepsilon_{1}^{2}(x)} & \geq \int_{1-2 \varepsilon_{1}(x)}^{1} \beta^{T}(y ; n-k, k) d y \\
& >\int_{1-2 \varepsilon_{1}(x)}^{1-\varepsilon_{1}(x)} \beta^{T}(y ; n-k, k) d y>A_{1}(x) \int_{1-2 \varepsilon_{1}(x)}^{1-\varepsilon_{1}(x)} d y=A_{1}(x) \varepsilon_{1}(x) .
\end{aligned}
$$

\footnotetext{
${ }^{31}$ The cases $y(x)=0$ and/or $\bar{y}(x) \leq 1$ are similar.

${ }^{32}$ Note that the mode of $\beta^{T}(\cdot)$ is less than $1-2 \varepsilon_{1}(x)$.
} 
Therefore,

$$
A_{1}(x)<\frac{\operatorname{Var}_{\beta^{T}}}{4 \varepsilon_{1}^{3}(x)}
$$

Denote

$$
A_{2}(x)=\int_{1-\varepsilon_{1}(x)}^{1}|\Upsilon(x, y)| d y .
$$

Thus, noting that $A_{1}(x)>\beta^{T}(y ; n-k, k)$ for $1-\varepsilon_{1}(x)<y<1$,

$$
\left|\int_{1-\varepsilon_{1}(x)}^{1} \Upsilon(x, y) \beta^{T}(y ; n-k, k) d y\right|<A_{1}(x) \int_{1-\varepsilon_{1}(x)}^{1}|\Upsilon(x, y)| d y<A_{2}(x) \frac{\operatorname{Var}_{\beta^{T}}}{4 \varepsilon_{1}^{3}(x)} .
$$

Now consider the second term in (32). By Taylor's expansion $\Upsilon(x, y)=\Upsilon\left(x, E_{\beta^{T}}\right)+$ $\frac{\partial \Upsilon\left(x, E_{\left.\beta^{T}\right)}\right)}{\partial y}\left(y-E_{\beta^{T}}\right)+\frac{1}{2} \frac{\partial^{2} \Upsilon\left(x, y^{*}\right)}{\partial y}\left(y-E_{\beta^{T}}\right)^{2}$, where $y^{*}$ is between $y$ and $E_{\beta^{T}}$. (Note that $y^{*}$ depends upon $y$.)

$$
\begin{aligned}
& \left|\int_{\underline{y}(x)}^{1-\varepsilon_{1}(x)} \Upsilon(x, y) \beta^{T}(y ; n-k, k) d y-\Upsilon\left(x, E_{\beta^{T}}\right)\right| \\
= & \mid \int_{\underline{y}(x)}^{1-\varepsilon_{1}(x)}\left(\Upsilon\left(x, E_{\beta^{T}}\right)+\frac{\partial \Upsilon\left(x, E_{\beta^{T}}\right)}{\partial y}\left(y-E_{\beta^{T}}\right)+\frac{1}{2} \frac{\partial^{2} \Upsilon\left(x, y^{*}\right)}{\partial y}\left(y-E_{\beta^{T}}\right)^{2}\right) \beta^{T}(y ; n-k, k) d y \\
\leq & \quad \int_{\underline{y}(x)}^{1-\varepsilon_{1}(x)} \Upsilon\left(x, E_{\beta^{T}}\right) \mid \\
& +\left|\int_{\underline{y}(x)}^{1-\varepsilon_{1}(x)} \frac{\partial \Upsilon\left(x, E_{\beta^{T}}\right)}{\partial y}\left(y-E_{\beta^{T}}\right) \beta^{T}(y ; n-k, k) d y-\Upsilon\left(x, E_{\beta^{T}}\right)\right| \\
& +\left|\int_{\underline{y}(x)}^{1-\varepsilon_{1}(x)} \frac{1}{2} \frac{\partial^{2} \Upsilon\left(x, y^{*}\right)}{\partial y^{2}}\left(y-E_{\beta^{T}}\right)^{2} \beta^{T}(y ; n-k, k) d y\right|
\end{aligned}
$$

By definition of $E_{\beta^{T}}, \int_{\underline{y}(x)}^{1} \frac{\partial \Upsilon\left(x, E_{\beta^{T}}\right)}{\partial y}\left(y-E_{\beta^{T}}\right) \beta^{T}(y, n-k, k) d y=0$, and therefore

$$
\int_{\underline{y}(x)}^{1-\varepsilon_{1}(x)} \frac{\partial \Upsilon\left(x, E_{\beta^{T}}\right)}{\partial y}\left(y-E_{\beta^{T}}\right) \beta^{T}(y ; n-k, k) d y=-\int_{1-\varepsilon_{1}(x)}^{1} \frac{\partial \Upsilon\left(x, E_{\beta^{T}}\right)}{\partial y}\left(y-E_{\beta^{T}}\right) \beta^{T}(y ; n-k, k) d y .
$$

Similarly,

$$
\int_{\underline{y}(x)}^{1-\varepsilon_{1}(x)} \Upsilon\left(x, E_{\beta^{T}}\right) \beta^{T}(y ; n-k, k) d y-\Upsilon\left(x, E_{\beta^{T}}\right)=-\int_{1-\varepsilon_{1}(x)}^{1} \Upsilon\left(x, E_{\beta^{T}}\right) \beta^{T}(y ; n-k, k) d y .
$$


Then, substituting these inside the absolute values of (34) yields

$$
\begin{aligned}
& \left|\int_{\underline{y}(x)}^{1-\varepsilon_{1}(x)} \Upsilon(x, y) \beta^{T}(y ; n-k, k) d y-\Upsilon\left(x, E_{\beta^{T}}\right)\right| \\
& \leq\left|\int_{1-\varepsilon_{1}(x)}^{1} \Upsilon\left(x, E_{\beta^{T}}\right) \beta^{T}(y ; n-k, k) d y\right|+\left|\int_{1-\varepsilon_{1}(x)}^{1} \frac{\partial \Upsilon\left(x, E_{\beta^{T}}\right)}{\partial y}\left(y-E_{\beta^{T}}\right) \beta^{T}(y ; n-k, k) d y\right| \\
& \quad+\left|\int_{\underline{y}(x)}^{1-\varepsilon_{1}(x)} \frac{1}{2} \frac{\partial^{2} \Upsilon\left(x, y^{*}\right)}{\partial y^{2}}\left(y-E_{\beta^{T}}\right)^{2} \beta^{T}(y ; n-k, k) d y\right| .
\end{aligned}
$$

Recall that $E_{\beta^{T}}<1-4 \varepsilon_{1}(x)$, and both $\Upsilon(x, y)$ and $\frac{\partial \Upsilon(x, y)}{\partial y}$ are bounded for $\underline{y}(x) \leq y \leq$ $1-\varepsilon_{1}(x)$. Therefore, there exists $A_{3}(x)$, continuous in $x$, such that

$$
\left|\Upsilon\left(x, E_{\beta^{T}}\right)\right|+\left|\frac{\partial \Upsilon\left(x, E_{\beta^{T}}\right)}{\partial y}\left(1-E_{\beta^{T}}\right)\right|<A_{3}(x) .
$$

Then by Chebyshev's inequality

$$
\left|\int_{1-\varepsilon_{1}(x)}^{1} \Upsilon\left(x, E_{\beta^{T}}\right) \beta^{T}(y ; n-k, k) d y\right|+\left|\int_{1-\varepsilon_{1}(x)}^{1} \frac{\partial \Upsilon\left(x, E_{\beta^{T}}\right)}{\partial y}\left(y-E_{\beta^{T}}\right) \beta^{T}(y ; n-k, k) d y\right|<A_{3}(x) \frac{\operatorname{Var}_{\beta^{T}}}{\varepsilon_{1}^{2}(x)} .
$$

Finally, denote

$$
A_{4}(x)=\max _{t \in\left[\underline{y}(x), 1-\varepsilon_{1}(x)\right]}\left|\frac{\partial^{2} \Upsilon(x, t)}{\partial y^{2}}\right| .
$$

Note that $A_{4}(x)$ is also continuous in $x$. Then

$$
\left|\int_{\underline{y}(x)}^{1-\varepsilon_{1}(x)} \frac{1}{2} \frac{\partial^{2} \Upsilon\left(x, y^{*}\right)}{\partial y^{2}}\left(y-E_{\beta^{T}}\right)^{2} \beta^{T}(y ; n-k, k) d y\right|<A_{4}(x) \operatorname{Var}_{\beta^{T}} .
$$

Combining these developments provides a bound on (34):

$$
\left|\int_{\underline{y}(x)}^{1-\varepsilon_{1}(x)} \Upsilon(x, y) \beta^{T}(y ; n-k, k) d y-\Upsilon\left(x, E_{\beta^{T}}\right)\right| \leq A_{3}(x) \frac{\operatorname{Var}_{\beta^{T}}}{\varepsilon_{1}^{2}(x)}+A_{4}(x) \operatorname{Var}_{\beta^{T}} .
$$

Substituting (33) and (35) into (32), recalling that $\operatorname{Var}_{\beta^{T}}<\frac{1}{n}$, and denoting $C_{1}(x)=$ $\frac{A_{3}(x)}{\varepsilon_{1}^{2}(x)}+\frac{A_{2}(x)}{4 \varepsilon_{1}^{3}(x)}+A_{4}(x)$ yield

$$
\left|\int_{\underline{y}(x)}^{1} \Upsilon(x, y) \beta^{T}(y ; n-k, k) d y-\Upsilon\left(x, E_{\beta^{T}}\right)\right|<C_{1}(x) / n .
$$

It remains to substitute $y_{n k}$ for $E_{\beta^{T}}$. Recall that for a truncated beta distribution there exists $\theta$ such that $\left|E_{\beta^{T}}-y_{n k}\right|<\theta / n$, where $y_{n k}$ is given by (19). Denote by $C_{2}(x)=$ $\max _{t \in[\underline{y}(x), 1-\varepsilon]}\left|\frac{\partial \Upsilon(x, t)}{\partial y}\right|$. By first-order Taylor expansion

$$
\left|\Upsilon\left(x, E_{\beta^{T}}\right)-\Upsilon\left(x, y_{n k}\right)\right|<C_{2}(x)\left|E_{\beta^{T}}-y_{n k}\right|<C_{2}(x) \theta / n .
$$

Denoting $C(x)=C_{1}(x)+C_{2}(x) \theta$ completes the proof. 


\section{Appendix D: Asymptotic Extraction Proofs}

\subsection{Proof of Corollary 7.3.}

Substituting $f(x \mid v)=h(x-v), F(x \mid v)=H(x-v)$ and diffuse prior $g(v)$ into (2) yields

$$
b_{n_{i} k_{i}}(x)=\frac{\int_{\underline{v}}^{\bar{v}} v h^{2}(x-v) H^{n_{i}-k_{i}-1}(x-v)(1-H(x-v))^{k_{i}-1} d v}{\int_{\underline{v}}^{\bar{v}} h^{2}(x-v) H^{n_{i}-k_{i}-1}(x-v)(1-H(x-v))^{k_{i}-1} d v} .
$$

Introducing the change of variables $t=x-v$ yields

$$
\begin{aligned}
b_{n_{i} k_{i}}(x) & =\frac{\int_{-\infty}^{\infty}(x-t) h^{2}(t) H^{n_{i}-k_{i}-1}(t)(1-H(t))^{k_{i}-1} d t}{\int_{-\infty}^{\infty} h^{2}(t) H^{n_{i}-k_{i}-1}(t)(1-H(t))^{k_{i}-1} d t} \\
& =x-\frac{\int_{-\infty}^{\infty} t h^{2}(t) H^{n_{i}-k_{i}-1}(t)(1-H(t))^{k_{i}-1} d t}{\int_{-\infty}^{\infty} h^{2}(t) H^{n_{i}-k_{i}-1}(t)(1-H(t))^{k_{i}-1} d t} .
\end{aligned}
$$

Next, denote

$$
\begin{aligned}
\alpha_{i} & =\frac{\left(n_{i}-1\right) !}{\left(k_{i}-1\right) !\left(n_{i}-k_{i}-1\right) !} \int_{-\infty}^{\infty} t H^{n_{i}-k_{i}-1}(t)(1-H(t))^{k_{i}-1} h^{2}(t) d t \\
\psi_{i} & =\frac{\left(n_{i}-1\right) !}{\left(k_{i}-1\right) !\left(n_{i}-k_{i}-1\right) !} \int_{-\infty}^{\infty} H^{n_{i}-k_{i}-1}(t)(1-H(t))^{k_{i}-1} h^{2}(t) d t .
\end{aligned}
$$

Note that $w_{i}(x)$ in (3) can be expressed via $\psi_{i}$ in $(37)$ as $w_{i}(x)=\frac{\pi_{i} n_{i} \psi_{i}}{\sum_{j=1}^{M} \pi_{j} n_{j} \psi_{j}}$, which does not depend on $x$ due to location invariance. Substituting into (36) yields $b_{n_{i} k_{i}}(x)=x-\alpha_{i} / \psi_{i}$, and substituting this formula, $\alpha_{i}$, and $\psi_{i}$ into (4) gives

$$
b^{*}(x)=\sum_{i=1}^{M} \frac{\pi_{i} n_{i} \psi_{i}}{\sum_{j=1}^{M} \pi_{j} n_{j} \psi_{j}} b_{n_{i} k_{i}}(x)=\frac{\sum_{i=1}^{M} \pi_{i} n_{i} \psi_{i}\left(x-\frac{\alpha_{i}}{\psi_{i}}\right)}{\sum_{j=1}^{M} \pi_{j} n_{j} \psi_{j}}=x-\frac{\sum_{i=1}^{M} \pi_{i} n_{i} \alpha_{i}}{\sum_{j=1}^{M} \pi_{j} n_{j} \psi_{j}} .
$$

\subsection{Proof of Theorem 7.4.}

In preparation of the proof, two lemmas that relate to the counterfactual sequence ${ }^{33}\left\{\mathcal{A}_{r}\right\}$ of auctions (in which the number of bidders is revealed prior to bid submission) are introduced first. Total expected profit in these auctions is

$$
S_{R}\left(\Omega_{\mathbf{k}_{r}}\right)=\sum_{i=1}^{M} \pi_{i} k_{i_{r}} E\left[V-P_{i_{r}}^{R}\right]
$$

\footnotetext{
${ }^{33}$ Defined in the proof of Theorem 4.2 .
} 
where $P_{i_{r}}^{R}$ denotes the corresponding auction price (conditional on the realization of the $i^{\text {th }}$ element of $\Omega_{\mathbf{k}_{r}}$ ), i.e., if bids were submitted after the number of bidders became known. Lemma 10.1 relates the profits when bids are submitted before and after the number of bidders becomes known. Lemma 10.2 shows that the latter profit, $S_{R}\left(\Omega_{\mathbf{k}_{r}}\right)$, is bounded. The proof of the theorem then combines these two lemmas.

Lemma 10.1 Let Assumptions 7.1 and 7.2 hold for a sequence of auctions $\left\{\mathcal{A}_{\zeta}\right\}_{\zeta=1,2, \ldots}$ and a corresponding counterfactual sequence of auctions $\left\{A_{r}\right\}$ with $\{r=\zeta\} \Rightarrow\left\{\Omega_{\mathbf{k}_{r}}=\Omega_{\mathbf{k}_{\zeta}}\right.$, $\left.\pi_{i_{r}}=\pi_{i_{\zeta}}, h_{r}(\cdot)=h_{\zeta}(\cdot)\right\}$, and with numbers of bidders revealed. There exists a function $D$ : $\Re_{+}^{M} \rightarrow \Re_{+}$such that

$$
S\left(\Omega_{\mathbf{k}_{\zeta}}\right)-S_{R}\left(\Omega_{\mathbf{k}_{\zeta}}\right)=\left(\sum_{i=1}^{M} \pi_{i} s_{i_{\zeta}} n_{i_{\zeta}}\right)\left(D\left(s_{1_{\zeta}}, \ldots, s_{M_{\zeta}}\right)+O\left(\frac{1}{n_{1_{\zeta}}}\right)\right),
$$

where $D\left(s_{1}, \ldots, s_{M}\right)=0$ if $s_{i}=s_{i \prime} \forall i, i^{\prime}$, and $D\left(s_{1}, \ldots, s_{M}\right)>0$ otherwise.

Proof. In $\mathcal{A}_{\zeta}$, let $J_{i}^{\zeta}$ denote the price-setting signal given the $i^{\text {th }}$ component of $\Omega_{\mathbf{k}_{\zeta}}$; this is the $\left(k_{i_{\zeta}}+1\right)^{\text {st }}$-highest of $n_{i_{\zeta}}$ signals. Since this is the same object in $\mathcal{A}_{r}$, we will use $J_{i}^{r}$ interchangeably with $J_{i}^{\zeta}$.

Given Assumptions 7.1 and 7.2, by Corollary 7.3, the price simplifies to

$$
P_{i}^{\zeta}=b_{\zeta}^{*}\left(J_{i}^{\zeta}\right)=J_{i}^{\zeta}-\frac{\sum_{i=1_{\zeta}}^{M_{\zeta}} \pi_{i} n_{i} \alpha_{i}}{\sum_{i=1_{\zeta}}^{M_{\zeta}} \pi_{i} n_{i} \psi_{i}}
$$

So total expected profit (eq. (6)) becomes

$$
S\left(\Omega_{\mathbf{k}_{\zeta}}\right)=\sum_{i=1_{\zeta}}^{M_{\zeta}} \pi_{i} k_{i} E\left[V-b_{\zeta}^{*}\left(J_{i}^{\zeta}\right)\right]=\sum_{i=1_{\zeta}}^{M_{\zeta}} \pi_{i} k_{i} E\left[V-J_{i}^{\zeta}\right]+\frac{\sum_{i=1_{\zeta}}^{M_{\zeta}} \pi_{i} n_{i} \alpha_{i}}{\sum_{i=1_{\zeta}}^{M_{\zeta}} \pi_{i} n_{i} \psi_{i}} \sum_{i=1_{\zeta}}^{M_{\zeta}} \pi_{i} k_{i} .
$$

With revealed numbers of bidders, the bid function is not $b^{*}$, but $b_{n_{i} k_{i}}$, since the $\left(n_{i}, k_{i}\right)$ pair is revealed before bids are submitted. So $P_{i}^{R}=b_{n_{i} k_{i}}\left(J_{i}\right)=x-\alpha_{i} / \psi_{i}$ and

$$
S_{R}\left(\Omega_{\mathbf{k}_{r}}\right)=\sum_{i=1_{r}}^{M_{r}} \pi_{i} k_{i} E\left[V-b_{i}\left(J_{i}^{\zeta}\right)\right]=\sum_{i=1_{r}}^{M_{r}} \pi_{i} k_{i} E\left[V-J_{i}^{\zeta}\right]+\sum_{i=1_{r}}^{M_{r}} \pi_{i} k_{i} \frac{\alpha_{i}}{\psi_{i}} .
$$

For $\zeta=r$, the difference $S\left(\Omega_{\mathbf{k}_{\zeta}}\right)-S_{R}\left(\Omega_{\mathbf{k}_{r}}\right)$ (how much seller would benefit could the numbers of bidders be revealed before bids were submitted):

$$
S\left(\Omega_{\mathbf{k}_{\zeta}}\right)-S_{R}\left(\Omega_{\mathbf{k}_{r}}\right)=\sum_{i=1_{\zeta}}^{M_{\zeta}} \pi_{i} k_{i} E\left[b_{n_{i} k_{i}}\left(J_{i}^{\zeta}\right)-b_{\zeta}^{*}\left(J_{i}^{\zeta}\right)\right]=\sum_{i=1_{\zeta}}^{M_{\zeta}} \pi_{i} k_{i}\left(\frac{\sum_{j=1_{\zeta}}^{M_{\zeta}} \pi_{j} n_{j} \alpha_{j}}{\sum_{j=1_{\zeta}}^{M_{\zeta}} \pi_{j} n_{j} \psi_{j}}-\frac{\alpha_{i}}{\psi_{i}}\right) .
$$


Introducing a change of variables $y=H(t)$, so $t=H^{-1}(y)$, the terms (37) can be rewritten as

$$
\begin{aligned}
\alpha_{i} & =\int_{0}^{1} H^{-1}(y) h\left(H^{-1}(y)\right) \beta\left(y ; n_{i}-k_{i}, k_{i}\right) d y, \\
\psi_{i} & =\int_{0}^{1} h\left(H^{-1}(y)\right) \beta\left(y ; n_{i}-k_{i}, k_{i}\right) d y,
\end{aligned}
$$

where $\beta\left(y ; n_{i}-k_{i}, k_{i}\right)$ is a pdf of beta-distribution. Applying Lemma 9.1 to $\alpha_{i}$ and $\psi_{i}$ in $(42)$ yields

$$
\begin{aligned}
& \alpha_{i}=h\left(H^{-1}\left(\frac{n_{i}-k_{i}}{n_{i}}\right)\right) H^{-1}\left(\frac{n_{i}-k_{i}}{n_{i}}\right)+O\left(\frac{1}{n_{i}}\right), \\
& \psi_{i}=h\left(H^{-1}\left(\frac{n_{i}-k_{i}}{n_{i}}\right)\right)+O\left(\frac{1}{n_{i}}\right) .
\end{aligned}
$$

Denoting

$$
\begin{aligned}
\rho(t) & =H^{-1}(1-t), \\
\psi^{0}(t) & =h\left(H^{-1}(1-t)\right),
\end{aligned}
$$

and using (43),

$$
\begin{aligned}
\frac{\alpha_{i}}{\psi_{i}} & =H^{-1}\left(1-s_{i}\right)+O\left(\frac{1}{n_{i}}\right)=\rho(s)+O\left(\frac{1}{n_{i}}\right), \\
\psi_{i} & =h\left(H^{-1}\left(1-s_{i}\right)\right)+O\left(\frac{1}{n_{i}}\right)=\psi^{0}(s)+O\left(\frac{1}{n_{i}}\right) .
\end{aligned}
$$

In this notation, (41) becomes ${ }^{34}$

$$
\begin{aligned}
S\left(\Omega_{\mathbf{k}_{\zeta}}\right)-S_{R}\left(\Omega_{\mathbf{k}_{r}}\right) & =\sum_{i=1_{\zeta}}^{M_{\zeta}} \pi_{i} n_{i} s_{i}\left(\frac{\sum_{j=1_{\zeta}}^{M_{\zeta}} \pi_{j} n_{j} \psi^{0}\left(s_{j}\right) \rho\left(s_{j}\right)}{\sum_{j=1}^{M_{\zeta}} \pi_{j} n_{j} \psi^{0}\left(s_{j}\right)}-\rho\left(s_{i}\right)+O\left(\frac{1}{n_{1}}\right)\right) \\
=\left(\sum_{i=1_{\zeta}}^{M_{\zeta}} \pi_{i} n_{i} s_{i}\right) & \left(\frac{\sum_{j=1_{\zeta}}^{M_{\zeta}} \pi_{j} n_{j} \psi^{0}\left(s_{j}\right) \rho\left(s_{j}\right)}{\sum_{j=1_{\zeta}}^{M_{\zeta}} \pi_{j} n_{j} \psi^{0}\left(s_{j}\right)}-\frac{\sum_{j=1_{\zeta}}^{M_{\zeta}} \pi_{j} n_{j} s_{j} \rho\left(s_{j}\right)}{\sum_{j=1_{\zeta}}^{M_{\zeta}} \pi_{j} n_{j} s_{j}}+O\left(\frac{1}{n_{1_{\zeta}}}\right)\right) .
\end{aligned}
$$

Define

$$
\lambda_{i}^{1}=\pi_{i} n_{i} \psi^{0}\left(s_{i}\right)>0, \lambda_{i}^{2}=\pi_{i} n_{i} s_{i}>0,
$$

and set

$$
D\left(s_{1}, \ldots, s_{M}\right)=\frac{\sum_{i=1}^{M} \lambda_{i}^{1} \rho\left(s_{i}\right)}{\sum_{i=1}^{M} \lambda_{i}^{1}}-\frac{\sum_{i=1}^{M} \lambda_{i}^{2} \rho\left(s_{i}\right)}{\sum_{i=1}^{M} \lambda_{i}^{2}} .
$$

\footnotetext{
${ }^{34}$ The first equality follows from the fact that $\sum \pi_{j} n_{j} \psi^{0}\left(s_{j}\right)$ in the denominator is positive. See also the last sentence of Footnote 26.
} 
It follows from (45) that $D$ satisfies (38). It remains to show that any $s_{i} \neq s_{j}$ implies $D\left(s_{1}, \ldots, s_{M}\right)>0$, and that $D\left(s_{1}, \ldots, s_{M}\right)=0$ implies $s_{i}=s_{j} \forall i, j$.

Note that $D\left(s_{1}, \ldots, s_{M}\right)$ is the difference of two weighted averages of $\rho\left(s_{i}\right)$. Furthermore,

$$
\left\{\rho\left(s_{i}\right)>\rho\left(s_{j}\right)\right\} \Leftrightarrow\left\{s_{i}<s_{j}\right\} \Leftrightarrow\left\{\frac{\lambda_{i}^{1}}{\lambda_{i}^{2}}>\frac{\lambda_{j}^{1}}{\lambda_{j}^{2}}\right\} \Leftrightarrow\left\{\frac{\lambda_{i}^{1}}{\lambda_{j}^{1}}>\frac{\lambda_{i}^{2}}{\lambda_{j}^{2}}\right\}
$$

because $\rho$ is decreasing by definition and because the ratio $\frac{\lambda_{i}^{1}}{\lambda_{i}^{2}}=\frac{\psi^{0}\left(s_{i}\right)}{s_{i}}$ is a decreasing function. To show the latter, introduce a change of variables $t=H^{-1}(1-s)$, so that $s=1-H(t)$. Then

$$
\frac{\psi^{0}(s)}{s}=\frac{h\left(H^{-1}(1-s)\right)}{s}=\frac{h(t)}{1-H(t)} .
$$

As Klemperer (1999) shows, an implication of SMLRP (eq. (1)) is that $\forall v, x$ such that $f(x \mid v)>0$ :

$$
\frac{\partial}{\partial v}\left(\frac{f(x \mid v)}{1-F(x \mid v)}\right)<0
$$

Therefore, since $f(x \mid v)=h(x-v)$ satisfies SMLRP, then for all $t$ such that $h(t)>0$ :

$$
\frac{d}{d t}\left(\frac{h(t)}{1-H(t)}\right)>0
$$

and $\psi^{0}(s) / s$ is decreasing in $s$.

From (47) it follows that the weight distribution with weights $\lambda_{i}^{1} / \sum_{j=1}^{n} \lambda_{j}^{1}$ places larger weights to larger $\rho\left(s_{i}\right)$ than the weight distribution with weights $\lambda_{i}^{2} / \sum_{j=1}^{n} \lambda_{j}^{2}$ does. Since all $\rho\left(s_{i}\right)>0, s_{i} \neq s_{j}$ implies $D\left(s_{1}, \ldots, s_{M}\right)>0$, and thus $D\left(s_{1}, \ldots, s_{M}\right)=0$ implies $s_{i}=s_{j} \forall i, j$.

Lemma 10.2 Let Assumptions 7.1 and 7.2 hold for a counterfactual sequence of auctions $\left\{A_{r}\right\}_{\zeta=1,2, \ldots}$. Then

$$
S_{R}\left(\Omega_{\mathbf{k}_{r}}\right)=\left(\sum_{i=1_{r}}^{M_{r}} \pi_{i} s_{i_{r}} n_{i_{r}}\right) O\left(\frac{1}{n_{1_{r}}}\right) .
$$

Proof. Throughout the proof the subscript $r$ is omitted, wherever no confusion is possible.

Recall $J_{i}$ is the price-setting signal given the $i^{\text {th }}$ component of $\Omega_{\mathbf{k}}$. Location invariance implies that $E\left[V-J_{i} \mid V=v\right]=E\left[V-J_{i}\right]$, i.e., does not depend on $v$ :

$$
E\left[V-J_{i}\right]=\frac{n_{i} !}{k_{i} !\left(n_{i}-k_{i}-1\right) !} \int_{-\infty}^{\infty}(v-x) H^{n_{i}-k_{i}}(x-v)(1-H(x-v))^{k_{i}} h(x-v) d x .
$$

Introducing the change of variables $t=x-v$ yields

$$
E\left[V-J_{i}\right]=-\frac{n_{i} !}{k_{i} !\left(n_{i}-k_{i}-1\right) !} \int_{-\infty}^{\infty} t H^{n_{i}-k_{i}}(t)(1-H(t))^{k_{i}} h(t) d t .
$$


Introducing a change of variables $y=H(t)$, so $t=H^{-1}(y)$, yields

$$
E\left[V-J_{i}\right]=-\int_{0}^{1} H^{-1}(y) \beta\left(n_{i}-k_{i}, k_{i}+1, y\right) d y .
$$

Applying Lemma 9.1 yields

$$
\begin{aligned}
E\left[V-J_{i}\right] & =-H^{-1}\left(\frac{n_{i}-k_{i}}{n_{i}+1}\right)+O\left(\frac{1}{n_{i}}\right) \\
& =-H^{-1}\left(\frac{n_{i}-k_{i}}{n_{i}}\right)+O\left(\frac{1}{n_{i}}\right),
\end{aligned}
$$

where the second equality holds since $\left(n_{i}-k_{i}\right) /\left(n_{i}+1\right)=\left[\left(n_{i}-k_{i}\right) / n_{i}\right]+O\left(1 / n_{i}\right)$.

Reordering equation (40) yields

$$
\begin{aligned}
S_{R}\left(\Omega_{\mathbf{k}_{r}}\right) & =\sum_{i=1_{r}}^{M_{r}} \pi_{i} k_{i} \frac{\alpha_{i}}{\psi_{i}}+\sum_{i=1_{r}}^{M_{r}} \pi_{i} k_{i} E\left[V-J_{i}^{\zeta}\right] \\
& =\sum_{i=1_{r}}^{M_{r}} \pi_{i} k_{i}\left(H^{-1}\left(\frac{n_{i}-k_{i}}{n_{i}}\right)-H^{-1}\left(\frac{n_{i}-k_{i}}{n_{i}}\right)+O\left(\frac{1}{n_{i}}\right)\right) \\
& =\left(\sum_{i=1_{r}}^{M_{r}} \pi_{i} n_{i} s_{i}\right) O\left(\frac{1}{n_{1_{r}}}\right),
\end{aligned}
$$

where the substitution for $E\left[V-J_{i}^{\zeta}\right]$ comes from (49), and for $\alpha_{i} / \psi_{i}$ from (44).

\section{Proof of Theorem 7.4.}

Combining (38) and (48),

$$
S\left(\Omega_{\mathbf{k}_{\zeta}}\right)=\left(\sum_{i=1_{\zeta}}^{M_{\zeta}} \pi_{i} s_{i} n_{i}\right)\left(D\left(s_{1_{\zeta}}, \ldots, s_{M_{\zeta}}\right)+O\left(\frac{1}{n_{1_{\zeta}}}\right)\right) .
$$

since $\zeta=r$ and $O\left(1 / n_{1_{\zeta}}\right)+O\left(1 / n_{1_{r}}\right)=O\left(1 / n_{1_{\zeta}}\right)$. To show $[i]$, note that $\left|\left(s_{i_{\zeta}}-s_{0}\right) n_{1_{\zeta}}\right|<$ $\omega \forall i, \zeta$ implies $s_{i_{\zeta}}=s_{0}+O\left(\frac{1}{n_{\zeta}}\right) \forall i$. Also note and that $D\left(s_{1}, \ldots, s_{M}\right)$ has a second-order Taylor expansion around $\left(s_{0}, \ldots, s_{0}\right)$ since $h$ (and thus $H, H^{-1}$, and $D$ ) is continuously twice differentiable by model assumption. Thus,

$$
\begin{aligned}
D\left(s_{1_{\zeta}}, \ldots, s_{M_{\zeta}}\right) & =D\left(s_{0}, \ldots, s_{0}\right)+\sum_{i=1}^{M} \frac{\partial}{\partial s_{i}} D\left(s_{0}, \ldots, s_{0}\right)\left(s_{i_{\zeta}}-s_{0}\right)+o\left(s_{i_{\zeta}}-s_{0}\right) \\
& =0+\sum_{i=1}^{M} \frac{\partial}{\partial s_{i}} D\left(s_{0}, \ldots, s_{0}\right) O\left(\frac{1}{n_{1_{\zeta}}}\right)+o\left(\frac{1}{n_{1_{\zeta}}}\right) \\
& =O\left(\frac{1}{n_{1_{\zeta}}}\right) .
\end{aligned}
$$


Thus, recalling that by assumption in $[i], n_{i_{\zeta}} / n_{1_{\zeta}} \leq n_{M_{\zeta}} / n_{1_{\zeta}} \leq \mu$,

$$
S\left(\Omega_{\mathbf{k}_{\zeta}}\right)=\left(\sum_{i=1_{\zeta}}^{M_{\zeta}} \pi_{i} s_{i} n_{i}\right)\left(O\left(\frac{1}{n_{1_{\zeta}}}\right)\right) \leq\left(\sum_{i=1_{\zeta}}^{M_{\zeta}} \pi_{i} s_{i}\right) \mu O(1),
$$

and, in particular, (7) holds.

To show $[i i]$, first note that it suffices to prove $\lim \sup _{\zeta^{\prime}} S\left(\Omega_{\mathbf{k}_{\zeta^{\prime}}}\right)=\infty$, i.e., without loss of generality we may assume $\left\{\mathcal{A}_{\zeta^{\prime}}\right\}=\left\{\mathcal{A}_{\zeta}\right\}$. Since $s_{L} \neq s_{U}$ then, by Lemma 10.1, $D\left(s_{1}, \ldots, s_{M}\right)>0$. Then there exists $\zeta_{0}$ such that

$$
\left\{\zeta>\zeta_{1}\right\} \Rightarrow S\left(\Omega_{\mathbf{k}_{\zeta}}\right)>\left(\sum_{i=1_{\zeta}}^{M_{\zeta}} \pi_{i} s_{i} n_{i}\right)\left(D\left(s_{1}, \ldots, s_{M}\right) / 2\right) .
$$

Thus, since $n_{i_{\zeta}} \rightarrow \infty$, (8) holds. 


\section{References}

David, H.A., 1981. Order Statistics. John Wiley \& Sons, NY.

Harstad, R.M., Kagel, J., Levin, D., 1990. Equilibrium bid functions for auctions with an uncertain number of bidders. Econ. Letters 33, 35-40.

Hong, H., Shum, M., 2004. Rates of information aggregation in common value auctions. J. Econ. Theory 116, 1-40.

Jackson, M.O., Kremer, I., 2005. The relevance of a choice of auction format in a competitive environment. Working paper. California Institute of Technology and Stanford University.

Klemperer, P., 1999. Auction theory: A guide to the literature. J. Econ. Surveys 13, 227-286.

Kremer, I., 2002. Information aggregation in common value auctions. Econometrica 70, 1675-1682.

Matthews, S.A., 1987. Comparing auctions for risk averse buyers: A buyer's point of view. Econometrica 55, 633-646.

Milgrom, P.R., 1979. A convergence theorem for competitive bidding with differential information. Econometrica 47, 679-688.

Milgrom, P.R., 1981. Rational expectations, information acquisition, and competitive bidding. Econometrica 49, 921-943.

Milgrom, P.R., Weber, R.J., 1982. A theory of auctions and competitive bidding. Econometrica 50, 1089-1122.

Pesendorfer, W., Swinkels, J.M., 1997. The loser's curse and information aggregation in common value auctions. Econometrica 65, 1247-1281.

Riley, J.G., 1988. Ex post information in auctions. Rev. Econ. Stud. 55, 409-429.

Wilson, R.W., 1977. A bidding model of perfect competition. Rev. Econ. Stud. 44, 511-518.

Winkler, R.L., Brooks, D.G., 1980 Competitive bidding with dependent value estimates. Operations Research 28, 603-613. 